\title{
Small vertical vibrations of strings with moving ends
}

\author{
Tania Nunes Rabello, María Cristina Campos Vieira, \\ Cicero Lopes Frota and Luis Adauto Medeiros
}

\author{
Dep. Matemática - ITA, São José dos Campos, SP, Brasil, tania@ita.br \\ Dep. Matemática - ITA, São José dos Campos, SP, Brasil, cristina@ita.br \\ Dep. Matemática - UEM, Maringá, PR, clfrota@uem.br \\ IM-UFRJ, Rio de Janeiro, RJ, Brasil, Imedeiros@abc.org.br
}

Recibido: 17 de Enero de 2002

Aceptado: 23 de Septiembre de 2002

Dedicated to the memory of Jacques-Louis Lions

\begin{abstract}
In this work we investigate a mathematical model for small vertical vibrations of a stretched string when the ends vary with the time $t$ and the cross sections of the string is variable and the density of the material is also variable, that is, $\rho=\rho(x)$. It contains Kirchhoff model for fixed ends. We obtain solutions by Galerkin method and estimates in Sobolev spaces.
\end{abstract}

2000 Mathematics Subject Classification: 74H45.

Key words: Strings, Kirchhoff, Sobolev, Galerkin, vibrations.

\section{Introduction}

In the present article we investigate, from the point of view of mathematical analysis, a model for small vertical vibration of a stretched elastic string when the ends of the string are variables and its material is not homogeneous. In order to make clear the hypothesis we, first of all, deduce the model.

In fact, let us consider a Cartesian orthogonal system of coordinates in $\mathbb{R}^{2}$, $(x, 0, u)$. We suppose the string on the ordinate $0 x$, with ends $0<\alpha_{0}<\beta_{0}$ and suppose $\alpha_{0}, \beta_{0}$ has small displacements $0<\alpha(t)<\alpha_{0}<\beta_{0}<\beta(t)$. Thus, at time $t$, the lenght of the string is $[\alpha(t), \beta(t)]$ which is a deformation of the rest position $\left[\alpha_{0}, \beta_{0}\right]$. The vertical displacement of the point $x \in[\alpha(t), \beta(t)]$, at time $t$, is represented, in $\mathbb{R}^{2}$, 
by $u(x, t)$. The problem is to find a mathematical model for the small displacements $u(x, t)$.

We suppose $u(x, t)$ twice continuously derivable with respect to $x$ and $t$. The hypothesis of small deformations means $\left|\frac{\partial u}{\partial x}\right| \ll 1$, i.e. the gradient of deformations $u(x, t)$ is small.

Let us denote by $\tau_{0}$ the initial tension of the string at the rest position $\left[\alpha_{0}, \beta_{0}\right]$; by $\hat{\tau}(t)$ the tension of the string in the position $[\alpha(t), \beta(t)]$ which is the deformation of $\left[\alpha_{0}, \beta_{0}\right]$ and by $\tau(t)$ the tension of the curve deformation $u(x, t)$ of $[\alpha(t), \beta(t)]$. The tension at each point of the curve $u(x, t)$ is a vector that has the direction of the tangent vector of this curve at this point and has modulus $\tau(t)$. Thus its vertical component is

$$
\tau(t) \sin \theta
$$

where $\theta$ is the angle of the direction $0 x$ with the tangent vector. From the hypothesis of small deformations we don't consider the horizontal component of the tension. We have $\sin \theta \approx \operatorname{tg} \theta=\frac{\partial u}{\partial x}$. Thus the vertical component of $\overrightarrow{\tau(t)}$ is

$$
\tau(t) \sin \theta=\tau(t) \operatorname{tg} \theta=\tau(t) \frac{\partial u}{\partial x} .
$$

The variation of the tension generates a force and by second Newton's Law, we obtain

$$
\frac{\partial}{\partial x}(\tau(t) \sin \theta)=m \frac{\partial^{2} u}{\partial t^{2}},
$$

where $\frac{\partial^{2} u}{\partial t^{2}}$ is the acceleration of the deformation $u(x, t)$ and $m$ is the mass of the string. We have

$$
m \frac{\partial^{2} u}{\partial t^{2}}=\tau(t) \frac{\partial^{2} u}{\partial x^{2}}
$$

Calculus of the Tension $\tau(t)$.

We calculate the tension $\tau(t)$ in function of $\alpha(t), \beta(t), u(x, t)$. In fact, by Hooke's Law, we have

$$
\hat{\tau}(t)-\tau_{0}=k \frac{\gamma(t)-\gamma_{0}}{\gamma_{0}},
$$

where $\gamma(t)=\beta(t)-\alpha(t)$ is the lenght of $[\alpha(t), \beta(t)]$ and $\gamma_{0}=\gamma(0)=\beta_{0}-\alpha_{0}$ the lenght of $\left[\alpha_{0}, \beta_{0}\right]$.

If we represent by $S(t)$ the lenght of the arc of the curve $u(x, t)$, deformation of $[\alpha(t), \beta(t)]$, we obtain:

$$
S(t)=\int_{\alpha(t)}^{\beta(t)} \sqrt{1+\left(\frac{\partial u}{\partial x}\right)^{2}} d x \approx \int_{\alpha(t)}^{\beta(t)}\left(1+\frac{1}{2}\left(\frac{\partial u}{\partial x}\right)^{2}\right) d x
$$


By hypothesis of small deformations $\left|\frac{\partial u}{\partial x}\right| \ll 1$. Thus

$$
S(t)-\gamma(t)=\frac{1}{2} \int_{\alpha(t)}^{\beta(t)}\left(\frac{\partial u}{\partial x}\right)^{2} d x
$$

By Hooke's Law we obtain

$$
\tau(t)-\hat{\tau}(t)=k \frac{S(t)-\gamma(t)}{\gamma(t)}
$$

or

$$
\tau(t)-\hat{\tau}(t)=\frac{k}{2 \gamma(t)} \int_{\alpha(t)}^{\beta(t)}\left(\frac{\partial u}{\partial x}\right)^{2} d x
$$

From (1.2) and (1.3) we obtain the tension $\tau(t)$

$$
\tau(t)=\tau_{0}+k \frac{\gamma(t)-\gamma_{0}}{\gamma_{0}}+\frac{1}{2 \gamma(t)} \int_{\alpha(t)}^{\beta(t)}\left(\frac{\partial u}{\partial x}\right)^{2} d x
$$

Substituting $\tau(t)$ given by (1.4) in (1.1) and dividing by $m$, we obtain

$$
\frac{\partial^{2} u}{\partial t^{2}}-\left(\frac{\tau_{0}}{m}+\frac{k}{m} \frac{\gamma(t)-\gamma_{0}}{\gamma_{0}}+\frac{k}{2 m \gamma(t)} \int_{\alpha(t)}^{\beta(t)}\left(\frac{\partial u}{\partial x}\right)^{2} d x\right) \frac{\partial^{2} u}{\partial x^{2}}=0
$$

This is a model for small vertical deformations $u(x, t)$ when the ends of the string are not fixed, that is, it has small displacements $\alpha(t)<\alpha_{0}$ and $\beta(t)>\beta_{0}$.

Remark 1.1. If we suppose the extremes of the string fixed, that is, $\alpha(t)=\alpha_{0}$, $\beta(t)=\beta_{0}$ for all $t>0$. The model (1.5) reduces to

$$
\frac{\partial^{2} u}{\partial t^{2}}-\left(\frac{\tau_{0}}{m}+\frac{k}{2 m \gamma_{0}} \int_{\alpha_{0}}^{\beta_{0}}\left(\frac{\partial u}{\partial x}\right)^{2} d x\right) \frac{\partial^{2} u}{\partial x^{2}}=0
$$

proposed by Kirchhoff in 1883, (cf. Kirchhoff [11]). The mathematical aspects of this model were largely investigated. See, for example, Arosio-Spagnolo [1], Bernstein [3], Dickey [6], Hazoya-Yamada [9], Lions [14], Pohozhaev [21], [22], [23]. A survey of the results about the mathematical aspects of Kirchhoff model can be found in Medeiros-Limaco-Menezes [19], Part one. See also Milla Miranda-Jutuca [20] for non homogeneous boundary conditions. For explicit solutions in dimension one see Ebihara-Tanaka [7], for blow-up Bainov-Minchev [2].

Remark 1.2. When we suppose the tension constant, equal to $\tau_{0}$, we don't have the nonlinear contribution in (1.5) and it reduces to

$$
\frac{\partial^{2} u}{\partial t^{2}}-\frac{\tau_{0}}{m} \frac{\partial^{2} u}{\partial x^{2}}=0
$$


very well known as d'Alembert model (1743), (cf. Lüzen [17]).

Remark 1.3. For the case of variable ends it was investigated, initially, by LimacoMedeiros [13], Medeiros-Limaco-Menezes [19], Part two and Limaco-Medeiros [12], for membranes. For numerical experiments see I-Shih-Liu \& Rincon [10].

Remark 1.4. Note that in the model above $k=a E$ where $a$ is the area of the crosssection of the string and $E$ the Young's modulus of the material of the string. In the mathematical investigations above cited this cross-section supposed to be constant. The same for the density, that is, the mass per unity of lenght. In the present article we investigate, from the mathematical point of view, the model (1.5) when the crosssection depends on the ordinate $x$ and, consequently, the mass per unity of lenght depends also on $x$. In general, we will consider a mathematical model of the type

$$
\frac{\partial^{2} u}{\partial t^{2}}-\widehat{M}\left(x, t, \frac{1}{\gamma(t)} \int_{\alpha(t)}^{\beta(t)}\left(\frac{\partial u}{\partial x}\right)^{2} d x\right) \frac{\partial^{2} u}{\partial x^{2}}=\hat{f}(x, t),
$$

for $\alpha(t)<x<\beta(t), \quad t \geq 0$ and $\widehat{M}(x, t, \lambda)$ a function for $\alpha(t)<x<\beta(t), \quad t \geq 0$ and $0<\lambda<\infty$. Note that the models (1.5), (1.6) and (1.7) are particular cases of (1.8) when we specify $\widehat{M}(x, t, \lambda)$.

Rivera-Rodriguez [24] and Frota [8] investigated a mixed problem for (1.8) but they generalized only the Kirchhoff model (1.6) when $k=k(x)$ and the mass per unit of lenght depends on $x$ too. They did not considered the moving ends as will be done in the present investigation.

In Section 2 we will fixe the notations and the assumptions on $\widehat{M}(x, t, \lambda), \quad \alpha(t)$, $\beta(t), \quad \hat{f}(x, t)$ and formulate a mixed problem for (1.8). In the Section 3 we prove the theorems of Section 2. At the end of the article we have a small list of references.

\section{Notations and Statements of the Main Results}

We represent by $\widehat{Q}$ the noncylindrical domain of $\mathbb{R}^{2}$ defined as follows:

$$
\widehat{Q}=\left\{(x, t) \in \mathbb{R}^{2} \mid \alpha(t)<x<\beta(t), \text { for all } t \geq 0\right\} .
$$

The lateral boundary $\widehat{\Sigma}$ of $\widehat{Q}$ is defined by

$$
\widehat{\Sigma}=\bigcup_{0<t<T}\{(\alpha(t), \beta(t)) \times\{t\}\}
$$

for all $T>0$.

Let us represent by $\widehat{L}$ the operator acting on real functions $u(x, t)$ defined in $(x, t) \in \widehat{Q}$, by

$$
\widehat{L} u(x, t)=\frac{\partial^{2} u}{\partial t^{2}}-\widehat{M}\left(x, t, \frac{1}{\gamma(t)} \int_{\alpha(t)}^{\beta(t)}\left(\frac{\partial u}{\partial x}\right)^{2} d x\right) \frac{\partial^{2} u}{\partial x^{2}} .
$$


Thus, we formulate the mixed problem for $\widehat{L}$ in $\widehat{Q}$ as

$$
\mid \begin{array}{ll}
\widehat{L} u(x, t)=\hat{f}(x, t) & \text { in } \widehat{Q} \\
u(x, t)=0 & \text { for }(x, t) \in \widehat{\Sigma} \\
u(x, 0)=u_{0}(x), \frac{\partial u}{\partial t}(x, 0)=u_{1}(x) & \text { in } \alpha_{0}<x<\beta_{0} .
\end{array}
$$

We need hypotheses on $\widehat{M}(x, t, \lambda), \quad \alpha(t), \quad \beta(t)$ and $\hat{f}(x, t)$.

The notations and results on Sobolev spaces are contained in Lions-Magenes [16], Lions [15] and Brezis [4].

In fact, for $\Omega=(0,1)$ we consider the Hilbert spaces $L^{2}(\Omega)$ and $H_{0}^{1}(\Omega)$ with scalar product and norms denoted, respectively, by $(),,|\cdot|$ and $(()),\|\cdot\|)$ defined by

$$
\begin{aligned}
& (u, v)=\int_{0}^{1} u(y) v(y) d y, \quad|u|^{2}=\int_{0}^{1} u^{2}(y) d y, \text { for all } u, v \in L^{2}(0,1) \\
& ((u, v))=\int_{0}^{1} \frac{\partial u}{\partial y} \frac{\partial v}{\partial y} d y, \quad\|u\|^{2}=\int_{0}^{1}\left(\frac{\partial u}{\partial y}\right)^{2} d y, \text { for all } u, v \in H_{0}^{1}(0,1) .
\end{aligned}
$$

Note that

$$
H_{0}^{1}(0,1)=\left\{v \in L^{2}(0,1) ; \frac{\partial v}{\partial y} \in L^{2}(0,1), v(0)=v(1)=0\right\} .
$$

All derivatives are in the sense of distributions.

We also consider $\Omega_{t}=(\alpha(t), \beta(t))$ and the spaces $L^{2}\left(\Omega_{t}\right)$ and $H_{0}^{1}\left(\Omega_{t}\right)$.

We observe that when $\alpha(t)<x<\beta(t), \quad 0<t<T$, we have

$$
y=\frac{x-\alpha(t)}{\gamma(t)}, \quad 0<t<T
$$

and $0<y<1$. Thus, when $(x, t)$ varies in $\widehat{Q}$ the point $(y, t)$ varies in the cylinder $Q=(0,1) \times(0, T)$. Therefore, we define a mapping

$$
\phi: \widehat{Q} \rightarrow Q
$$

such that $\phi(x, t)=(y, t)$, with $y=\frac{x-\alpha(t)}{\gamma(t)}$.

In order to transform, by $\phi$, the operator $\widehat{L} u(x, t)$ to obtain an operator defined for functions $v(y, t)$, with $(y, t) \in Q$, we need certain assumptions.

We consider $\widehat{M}(x, t, \lambda)$ a numerical function defined for $x \in \Omega_{t}=(\alpha(t), \beta(t))$, $t \in(0, T)$ and $\lambda \in(0, K), \quad K$ any positive number. Suppose

$$
\widehat{M}(x, t, \lambda) \text { and } \frac{\partial \widehat{M}}{\partial \lambda}(x, t, \lambda)
$$

are continuous in $t, \quad \lambda$ and measurable in $x$. 


$$
\widehat{M}(x, t, \lambda), \quad \frac{\partial \widehat{M}}{\partial x}(x, t, \lambda), \quad \frac{\partial \widehat{M}}{\partial \lambda}(x, t, \lambda)
$$

belongs to $L^{\infty}\left(\Omega_{t} \times(0, T) \times(0, K)\right)$ for all $K>0$.

$$
\widehat{M}(x, t, \lambda) \geq m_{0}>0 \text { for all } x \in \Omega_{t},
$$

$t \in(0, T), \quad T>0, \quad \lambda \in(0, K)$ for all $K>0$.

$$
\alpha, \beta \in C^{2}([0, T] ; \mathbb{R}), \quad \alpha(t)<\alpha_{0}<\beta_{0}<\beta(t),
$$

any $T>0, \quad \alpha(0)=\alpha_{0}, \quad \beta(0)=\beta_{0}$,

$$
\alpha^{\prime}(t)<0, \quad \beta^{\prime}(t)>0 \text { for all } t \in[0, T] ;
$$

$\alpha^{\prime}(0)=0, \quad \beta^{\prime}(0)=0$. Set $\gamma(t)=\beta(t)-\alpha(t)$ and suppose

$$
\gamma^{\prime}(t) \leq \frac{\delta}{12} \gamma_{0},
$$

$\delta>0$ defined by $(2.5)$ and $\gamma_{0}=\gamma(0)$.

$$
\left|\alpha^{\prime \prime}(t)+\gamma^{\prime \prime}(t) y\right| \leq \frac{\left|\alpha^{\prime}(t)+\gamma^{\prime}(t) y\right|^{2}}{\gamma}, \quad 0 \leq y \leq 1, \text { for all } t \in[0, T] .
$$

Remark 2.1. We have $\alpha^{\prime}(t)<0$, and $0<y<1$. By the definition of $\delta$, see (2.5), we have $\delta<\frac{3 \sqrt{m_{0}}}{\gamma_{0}}$ what implies for all $t \geq 0$ and $0<y<1$

$$
\left|\alpha^{\prime}+\gamma^{\prime} y\right|<\frac{\sqrt{m_{0}}}{4}
$$

Now we will transform, by means of (2.2), the noncylindrical mixed problem (2.1) into a cylindrical mixed problem.

Set $v(y, t)=u(x, t)$, for $y=\frac{x-\alpha(t)}{\gamma(t)}$. With this change of variable the operator $\widehat{L} u(x, t)$ is transformed into $L v(y, t)$ defined by

$$
\begin{aligned}
L v(y, t) & =\frac{\partial^{2} v}{\partial t^{2}}-\frac{1}{\gamma^{2}}\left[M\left(y, t, \frac{1}{\gamma^{2}}\|v(t)\|^{2}\right)-\frac{m_{0}}{16}\right] \frac{\partial^{2} v}{\partial y^{2}}- \\
& -\frac{\partial}{\partial y}\left(a(y, t) \frac{\partial v}{\partial y}\right)+b(y, t) \frac{\partial v^{\prime}}{\partial y}+c(y, t) \frac{\partial v}{\partial y}
\end{aligned}
$$

for $(y, t) \in Q$.

Note that

- $M(y, t, \lambda)=\widehat{M}(\gamma y+\alpha, t, \lambda)$

- $a(y, t)=\frac{m_{0}}{16 \gamma^{2}}-\left(\frac{\alpha^{\prime}+\gamma^{\prime} y}{\gamma}\right)^{2}$

- $b(y, t)=-2\left(\frac{\alpha^{\prime}+\gamma^{\prime} y}{\gamma}\right)$ 
- $c(y, t)=-\left(\frac{\alpha^{\prime \prime}+\gamma^{\prime \prime} y}{\gamma}\right)$

The force $\hat{f}(x, t)$ is transformed into $f(y, t)$ given by $f(y, t)=\hat{f}(\gamma y+\alpha, t)$.

The initial data $v(y, 0)=u_{0}(\gamma y+\alpha)$. For the velocity $\frac{\partial v}{\partial t}$ we have

$$
\frac{\partial u}{\partial t}(x, t)=\frac{\partial v}{\partial y} \frac{\partial y}{\partial t}+\frac{\partial v}{\partial t}(y, t)
$$

Note that $\frac{\partial y}{\partial t}=-\frac{\alpha^{\prime}+\gamma^{\prime} y}{\gamma}$, which is zero for $t=0$, by (H2). Therefore

$$
\frac{\partial v}{\partial t}(y, 0)=\varphi_{1}(y) \quad \text { where } \quad \varphi_{1}(y)=u_{1}\left(\gamma_{0} y+\alpha_{0}\right) .
$$

Thus, the noncylindrical mixed problem is transformed into the cylindrical mixed problem

$$
\mid \begin{aligned}
& L v(y, t)=f(y, t) \text { in } Q \\
& v(0, t)=v(1, t)=0 \text { for } 0<t<T \\
& v(y, 0)=\varphi_{0}(y), \frac{\partial v}{\partial t}(y, 0)=\varphi_{1}(y) \text { for } 0<y<1 .
\end{aligned}
$$

Now we have all the objects to formulate the theorems.

We consider the bilinear, continuous and coercive form defined for all $\xi, \eta \in$ $H_{0}^{1}(0,1)$ by

$$
a(t, \xi, \eta)=\int_{0}^{1} a(y, t) \frac{\partial \xi}{\partial y} \frac{\partial \eta}{\partial y} d y
$$

Remark 2.2. Note that $M(y, t, \lambda)$ is defined in $(0,1) \times(0, T) \times(0, \infty)$ with real values such that

- It is continuous in $t, \lambda$ and measurable in $y$.

- $M(y, t, \lambda) \in C^{1}((0,1) \times(0, T) \times(0, K))$, for all $K>0$.

- $M(y, t, \lambda), \quad \frac{\partial M}{\partial y}(y, t, \lambda), \quad \frac{\partial M}{\partial \lambda}(y, t, \lambda)$ belongs to $L^{\infty}((0,1) \times(0, T) \times(0, K))$ for all $K>0$.

- $M(y, t, \lambda) \geq m_{0}$ for all $(y, t, \lambda) \in(0,1) \times(0, T) \times(0, K)$, for all $K>0$.

We consider the following constants that will appear in the proofs of the theorems 
to be given in the next section

$$
\mid \begin{aligned}
& K_{0}=\frac{16}{15 m_{0}}, \quad K_{1}=\frac{32}{15 m_{0} \gamma_{0}}\left(1+\frac{16+4 \sqrt{m_{0}}}{15 \pi^{2} m_{0}}\right), \\
& K_{2}=\|M\|_{L^{\infty}\left(Q \times\left(0,1 / \pi^{2} \gamma_{0}^{2}\right)\right)}=\|\widehat{M}\|_{L^{\infty}\left(\widehat{Q} \times\left(0,1 / \pi^{2} \gamma_{0}^{2}\right)\right)}, \\
& K_{3}=\frac{1}{2}\left(1+\frac{K_{2}}{\gamma_{0}^{2}}\right), \quad \theta_{0}=\left\|\frac{\partial M}{\partial t}(0)\right\|_{L^{\infty}(Q)}, \\
& \theta=\left(1+e^{\left(1+K_{0} \theta_{0}\right) T}\right), \\
& K_{4}=\frac{64}{15 \pi^{2} m_{0}}\left(K_{3}+1\right) \theta, \quad K_{5}=\left\|\frac{\partial M}{\partial \lambda}\right\|_{L^{\infty}\left(Q \times\left(0, K_{4}\right)\right)}, \\
& \delta=\operatorname{Min}\left(1, \frac{3 \sqrt{m_{0}}}{\gamma_{0}}, \frac{\log 2}{3 T\left(1+2 K_{1} K_{5} \theta\right)},\left[\frac{\log 2}{6 K_{1} K_{5} \theta T}\right]^{1 / 2}\right) \\
& K_{\delta}=K_{3} \delta^{2}+\delta
\end{aligned}
$$

Note that will use the notation

$$
|\cdot|_{0}, \quad\|\cdot\|_{0}, \quad|\cdot|_{t}, \quad\|\cdot\|_{t}
$$

to represent the norms in $L^{2}\left(\Omega_{0}\right), \quad H_{0}^{1}\left(\Omega_{0}\right), L^{2}\left(\Omega_{t}\right), \quad H_{0}^{1}\left(\Omega_{t}\right)$ where $\Omega_{0}=\left(\alpha_{0}, \beta_{0}\right)$, $\Omega_{t}=(\alpha(t), \beta(t))$.

Theorem 2.1. Supose $M(y, t, \lambda)$ as in Remark 2.2,

$$
\left\|\frac{\partial M}{\partial t}\right\|_{L^{\infty}\left((0,1) \times(0, T) \times\left(0, K_{4}\right)\right)} \leq \frac{\log 2}{3 K_{0} T}
$$

and (H1), (H2), (H3). Take $f \in L^{2}\left(0, T ; H_{0}^{1}(0,1)\right)$, for $T>0$. Then, given $\varphi_{0} \in$ $H_{0}^{1}(0,1) \cap H^{2}(0,1), \quad \varphi_{1} \in H_{0}^{1}(0,1)$ such that

$$
\left|\frac{\partial^{2} \varphi_{0}}{\partial y^{2}}\right|^{2}+\left\|\varphi_{1}\right\|^{2}+\frac{1}{\delta} \int_{0}^{T}\|f(t)\|^{2} d t<\delta^{2},
$$

$\delta$ given by (2.5), there exists a unique function $v: Q \rightarrow \mathbb{R}$ satisfying

$$
\begin{gathered}
v \in L^{\infty}\left(0, T ; H_{0}^{1}(0,1) \cap H^{2}(0,1)\right) \\
v^{\prime} \in L^{\infty}\left(0, T ; H_{0}^{1}(0,1)\right) \\
v^{\prime \prime} \in L^{2}\left(0, T ; L^{2}(0,1)\right)
\end{gathered}
$$

which is solution of (2.4). 
Theorem 2.2. Suppose $\widehat{M}(x, t, \lambda), \quad \alpha, \quad \beta$ satisfying $(\widehat{M} 1),(\widehat{M} 2),(\widehat{M} 3),(H 1),(H 2)$, (H3) and

$$
\text { Ess Sup }\left\{\left|\frac{\partial \widehat{M}}{\partial t}(x, t, \lambda)\right| ;(x, t) \in \widehat{Q}, \lambda \in\left(0,1 / \pi^{2} \gamma_{0}\right)\right\} \leq \frac{\log 2}{3 K_{0} T} .
$$

Then, given $u_{0} \in H_{0}^{1}\left(\Omega_{0}\right) \cap H^{2}\left(\Omega_{0}\right), \quad u_{1} \in H_{0}^{1}\left(\Omega_{0}\right), \quad \hat{f} \in L^{2}\left(0, T ; H_{0}^{1}\left(\Omega_{t}\right)\right)$ such that

$$
\gamma_{0}^{3}\left|\frac{\partial^{2} u_{0}}{\partial x^{2}}\right|_{0}^{2}+\gamma_{0}\left\|u_{1}\right\|_{0}^{2}+\frac{1}{\delta} \int_{0}^{T} \gamma\|\hat{f}(t)\|_{t}^{2} d t \leq \delta^{2}
$$

there exists only one function $u: \widehat{Q} \rightarrow \mathbb{R}$ satisfying:

$$
\begin{gathered}
u \in L^{\infty}\left(0, T ; H_{0}^{1}\left(\Omega_{t}\right) \cap H^{2}\left(\Omega_{t}\right)\right) \\
u^{\prime} \in L^{\infty}\left(0, T ; H_{0}^{1}\left(\Omega_{t}\right)\right) \\
u^{\prime \prime} \in L^{2}\left(0, T ; L^{2}\left(\Omega_{t}\right)\right)
\end{gathered}
$$

which is solution of the mixed problem (2.1).

\section{Proof of the Theorems}

Proof of Theorem 2.1. We will proof this theorem by Galerkin method. In the scheme for the method, in each step $\mu \in \mathbb{N}$, we consider the nonlinear term evaluated in the step $\mu-1$. Then, in each step, we have a linear approximate problem what is the key point to obtain a priori estimates.

Let $\left\{w_{\nu}, \lambda_{\nu}\right\}, \quad \nu \in \mathbb{N}$, be the solutions of the spectral problem $\left(\left(w_{\nu}, v\right)\right)=$ $\lambda_{\nu}\left(w_{\nu}, v\right)$, for all $v \in H_{0}^{1}(0,1)$. We consider $\left(w_{\nu}\right)_{\nu \in \mathbb{N}}$ orthonormal complete set in $L^{2}(0,1)$ and orthogonal in $H_{0}^{1}(0,1)$ and in $H_{0}^{1}(0,1) \cap H^{2}(0,1)$. We can calculate explicitly and obtain $w_{\nu}(x)=\sin \nu \pi x$ and $\lambda_{\nu}=(\nu \pi)^{2}, \nu=1,2, \ldots$ We represent by $V_{0}=\{0\}$ the null vector space and $V_{\mu}=\left[\left\{w_{1}, w_{2}, \ldots, w_{\mu}\right\}\right]$ the vector subspace of $H_{0}^{1}(0,1) \cap H^{2}(0,1)$ generated by the vectors $w_{1}, w_{2}, \ldots, w_{\mu}$. Thus if $v_{\mu}(t) \in V_{\mu}$ we have

$$
v_{\mu}(t)=\sum_{\nu=1}^{\mu} g_{\nu \mu}(t) w_{\nu} .
$$

Approximate Scheme. We define $v_{0}(t)=0$ for all $t \in[0, T]$. For each $\mu=1,2 \ldots$ we consider $v_{\mu}:\left[0, T_{\mu}\right] \rightarrow V_{\mu}$ the unique solution of the linear initial value problem

$$
\begin{aligned}
& \left(v_{\mu}^{\prime \prime}, w\right)-\frac{1}{\gamma^{2}}\left(\left[M\left(y, t, \frac{1}{\gamma^{2}}\left\|v_{\mu-1}(t)\right\|^{2}\right)-\frac{m_{0}}{16}\right] \frac{\partial^{2} v_{\mu}}{\partial y^{2}}, w\right)+ \\
& +a\left(t, v_{\mu}, w\right)+\left(b(t) \frac{\partial v_{\mu}^{\prime}}{\partial y}, w\right)+\left(c(t) \frac{\partial v_{\mu}}{\partial y}, w\right)=\left(f_{\mu}(t), w\right)
\end{aligned}
$$

for all $w \in V_{\mu}$. 


$$
v_{\mu}(0)=v_{0 \mu} \rightarrow \varphi_{0} \quad \text { in } \quad H_{0}^{1}(0,1) \cap H^{2}(0,1)
$$

$$
v_{\mu}^{\prime}(0)=v_{1 \mu} \rightarrow \varphi_{1} \quad \text { in } \quad H_{0}^{1}(0,1)
$$

where

$$
\begin{gathered}
T_{\mu}=\operatorname{Sup}\left\{\tau ; 0<\tau \leq T_{\mu-1} \text { and } v_{\mu}:[0, \tau] \rightarrow V_{\mu}\right. \\
\text { is solution of }(3.1),(3.2),(3.3)\}
\end{gathered}
$$

with

$$
f_{\mu}(t)=\sum_{\nu=1}^{\mu}\left(f(t), w_{\nu}\right) w_{\nu}, \quad \text { for } \quad 0<t \leq T
$$

\section{Estimate (1)}

$$
\begin{aligned}
& \text { Set } w=-\frac{\partial^{2} v_{\mu}^{\prime}}{\partial y^{2}} \text { in (3.1). We have } \\
& \frac{1}{2} \frac{d}{d t}\left\|v_{\mu}^{\prime}(t)\right\|^{2}+\int_{0}^{1} \frac{1}{\gamma^{2}}\left[M\left(y, t, \frac{1}{\gamma^{2}}\left\|v_{\mu-1}(t)\right\|^{2}\right)-\frac{m_{0}}{16}\right] \frac{\partial^{2} v_{\mu}}{\partial y^{2}} \frac{\partial^{2} v_{\mu}^{\prime}}{\partial y^{2}} d y+ \\
& +a\left(t, v_{\mu},-\frac{\partial^{2} v_{\mu}^{\prime}}{\partial y^{2}}\right)-\int_{0}^{1} b(y, t) \frac{\partial v_{\mu}^{\prime}}{\partial y} \frac{\partial^{2} v_{\mu}^{\prime}}{\partial y^{2}} d y- \\
& -\int_{0}^{1} c(y, t) \frac{\partial v_{\mu}}{\partial y} \frac{\partial^{2} v_{\mu}^{\prime}}{\partial y^{2}} d y=\left(\left(f_{\mu}(t), v_{\mu}^{\prime}(t)\right)\right)
\end{aligned}
$$

Remark 3.1. In order to avoid complicated notation, we represent $\alpha(t), \quad \beta(t)$, $\gamma(t)$, by $\alpha, \beta, \gamma$. 
Analysis of the Terms in (3.5) We have

- $\frac{1}{2} \frac{d}{d t} \int_{0}^{1} \frac{1}{\gamma^{2}}\left[M\left(y, t, \frac{1}{\gamma^{2}}\left\|v_{\mu-1}(t)\right\|^{2}\right)-\frac{m_{0}}{16}\right]\left(\frac{\partial^{2} v_{\mu}}{\partial y^{2}}(y, t)\right)^{2} d y=$ $=-\int_{0}^{1} \frac{\gamma^{\prime}}{\gamma^{3}}\left(M\left(y, t, \frac{1}{\gamma^{2}}\left\|v_{\mu-1}(t)\right\|^{2}\right)-\frac{m_{0}}{16}\right]\left(\frac{\partial^{2} v_{\mu}}{\partial y^{2}}(y, t)\right)^{2} d y+$ $+\frac{1}{2} \int_{0}^{1} \frac{1}{\gamma^{2}} \frac{\partial M}{\partial t}\left(y, t, \frac{1}{\gamma^{2}}\left\|v_{\mu-1}(t)\right\|^{2}\right)\left(\frac{\partial^{2} v_{\mu}}{\partial y^{2}}(y, t)\right)^{2} d y+$ $+\int_{0}^{1} \frac{1}{\gamma^{2}} \frac{\partial M}{\partial \lambda}\left(y, t, \frac{1}{\gamma^{2}}\left\|v_{\mu-1}(t)\right\|^{2}\right)\left[-\frac{\gamma^{\prime}}{\gamma^{3}}\left\|v_{\mu-1}(t)\right\|^{2}+\right.$ $\left.+\frac{1}{\gamma^{2}}\left(\left(v_{\mu-1}(t), v_{\mu-1}^{\prime}(t)\right)\right)\right]\left(\frac{\partial^{2} v_{\mu}}{\partial y^{2}}(y, t)\right)^{2} d y+$ $+\int_{0}^{1} \frac{1}{\gamma^{2}}\left[M\left(y, t, \frac{1}{\gamma^{2}}\left\|v_{\mu-1}(t)\right\|^{2}\right)-\frac{m_{0}}{16}\right] \frac{\partial^{2} v_{\mu}}{\partial y^{2}}(y, t) \cdot \frac{\partial^{2} v_{\mu}^{\prime}}{\partial y^{2}}(y, t) d y$.

- $-\int_{0}^{1} c(y, t) \frac{\partial v_{\mu}}{\partial y}(y, t) \frac{\partial^{2} v_{\mu}^{\prime}}{\partial y^{2}} d y=$ $=\left.\left(\frac{\alpha^{\prime \prime}+\gamma^{\prime \prime} y}{\gamma}\right) \frac{\partial v_{\mu}}{\partial y}(y, t) \frac{\partial v_{\mu}^{\prime}}{\partial y}(y, t)\right|_{y=0} ^{y=1}-\int_{0}^{1} \frac{\partial}{\partial y}\left[\left(\frac{\alpha^{\prime \prime}+\gamma^{\prime \prime} y}{\gamma}\right) \frac{\partial v_{\mu}}{\partial y}(y, t)\right] \frac{\partial v_{\mu}^{\prime}}{\partial y}(y, t) d y=$ $=\left.\left(\frac{\alpha^{\prime \prime}+\gamma^{\prime \prime} y}{\gamma}\right) \frac{\partial v_{\mu}}{\partial y}(y, t) \frac{\partial v_{\mu}^{\prime}}{\partial y}(y, t)\right|_{y=0} ^{y=1}-\int_{0}^{1} \frac{\partial}{\partial y}\left(\frac{\alpha^{\prime \prime}+\gamma^{\prime \prime} y}{\gamma}\right) \frac{\partial v_{\mu}}{\partial y} \frac{\partial v_{\mu}^{\prime}}{\partial y} d y-$ $-\int_{0}^{1}\left(\frac{\alpha^{\prime \prime}+\gamma^{\prime \prime} y}{\gamma}\right) \frac{\partial^{2} v_{\mu}}{\partial y^{2}}(y, t) \frac{\partial v_{\mu}^{\prime}}{\partial y}(y, t) d y=\frac{\beta^{\prime \prime}}{\gamma} \frac{\partial v_{\mu}}{\partial y}(1, t) \frac{\partial v_{\mu}^{\prime}}{\partial y}(1, t)-$ $-\frac{\alpha^{\prime \prime}}{\gamma} \frac{\partial v_{\mu}}{\partial y}(0, t) \frac{\partial v_{\mu}^{\prime}}{\partial y}(0, t)-\int_{0}^{1} \frac{\gamma^{\prime \prime}}{\gamma} \frac{\partial v_{\mu}}{\partial y}(y, t) \frac{\partial v_{\mu}^{\prime}}{\partial y}(y, t) d y-$

$-\int_{0}^{1}\left(\frac{\alpha^{\prime \prime}+\gamma^{\prime \prime} y}{\gamma}\right) \frac{\partial^{2} v_{\mu}}{\partial y^{2}}(y, t) \frac{\partial v_{\mu}^{\prime}}{\partial y}(y, t) d y$.

- $-\int_{0}^{1} b(y, t) \frac{\partial v_{\mu}^{\prime}}{\partial y}(y, t) \frac{\partial^{2} v_{\mu}^{\prime}}{\partial y^{2}}(y, t) d y=$ $=\int_{0}^{1} 2\left(\frac{\alpha^{\prime}+\gamma^{\prime} y}{\gamma}\right) \frac{\partial v_{\mu}^{\prime}}{\partial y} \frac{\partial^{2} v_{\mu}^{\prime}}{\partial y^{2}} d y=\int_{0}^{1}\left(\frac{\alpha^{\prime}+\gamma^{\prime} y}{\gamma}\right) \frac{\partial}{\partial y}\left(\frac{\partial v_{\mu}^{\prime}}{\partial y}(y, t)\right)^{2} d y=$ 


$$
\begin{aligned}
& =\left.\left(\frac{\alpha^{\prime}+\gamma^{\prime} y}{\gamma}\right)\left(\frac{\partial v_{\mu}^{\prime}}{\partial y}(y, t)\right)^{2}\right|_{y=0} ^{y=1}-\int_{0}^{1}\left(\frac{\partial v_{\mu}^{\prime}}{\partial y}\right)^{2} \frac{\partial}{\partial y}\left(\frac{\alpha^{\prime}+\gamma^{\prime} y}{\gamma}\right) d y= \\
& =\frac{\beta^{\prime}}{\gamma}\left(\frac{\partial v_{\mu}^{\prime}}{\partial y}(1, t)\right)^{2}-\frac{\alpha^{\prime}}{\gamma}\left(\frac{\partial v_{\mu}^{\prime}}{\partial y}(1, y)\right)^{2}-\frac{\gamma^{\prime}}{\gamma}\left\|v_{\mu}^{\prime}(t)\right\|^{2} . \\
& \text { - } a\left(t, v_{\mu},-\frac{\partial^{2} v_{\mu}^{\prime}}{\partial y^{2}}\right)=\int_{0}^{1} \frac{\partial}{\partial y}\left(a(y, t) \frac{\partial v_{\mu}}{\partial y}\right)\left(\frac{\partial^{2} v_{\mu}^{\prime}}{\partial y^{2}}\right) d y= \\
& =\int_{0}^{1} \frac{\partial a}{\partial y}(y, t) \frac{\partial v_{\mu}}{\partial y} \frac{\partial^{2} v_{\mu}^{\prime}}{\partial y^{2}} d y+\int_{0}^{1} a(y, t) \frac{\partial^{2} v_{\mu}}{\partial y^{2}} \frac{\partial^{2} v_{\mu}^{\prime}}{\partial y^{2}} d y= \\
& =\int_{0}^{1} b(y, t) \frac{\gamma^{\prime}}{\gamma} \frac{\partial v_{\mu}}{\partial y} \frac{\partial^{2} v_{\mu}^{\prime}}{\partial y^{2}} d y+ \\
& +\frac{1}{2} \frac{d}{d t} \int_{0}^{1} a(y, t)\left(\frac{\partial v_{\mu}}{\partial y}\right)^{2} d y-\int_{0}^{1} a^{\prime}(y, t)\left(\frac{\partial^{2} v_{\mu}}{\partial y^{2}}\right)^{2} d y= \\
& =-2 \frac{\beta^{\prime} \gamma^{\prime}}{\gamma^{2}} \frac{\partial v_{\mu}}{\partial y}(1, t) \frac{\partial v_{\mu}^{\prime}}{\partial y}(1, t)+2 \frac{\alpha^{\prime} \gamma^{\prime}}{\gamma^{2}} \frac{\partial v_{\mu}}{\partial y}(0, t) \frac{\partial v_{\mu}^{\prime}}{\partial y}(0, t)+ \\
& +\int_{0}^{1} 2\left(\frac{\gamma^{\prime}}{\gamma}\right) \frac{\partial v_{\mu}}{\partial y}(y, t) \frac{\partial v_{\mu}^{\prime}}{\partial y}(y, t) d y+ \\
& +\int_{0}^{1} 2\left(\frac{\alpha^{\prime}+\gamma^{\prime} y}{\gamma^{2}}\right) \gamma^{\prime} \frac{\partial^{2} v_{\mu}}{\partial y^{2}}(y, t) \frac{\partial v_{\mu}^{\prime}}{\partial y}(y, t) d y+ \\
& +\frac{1}{2} \frac{d}{d t} \int_{0}^{1} a(y, t)\left(\frac{\partial^{2} v_{\mu}}{\partial y^{2}}(y, t)\right)^{2} d y-\frac{1}{2} \int_{0}^{1} a^{\prime}(y, t)\left(\frac{\partial^{2} v_{\mu}}{\partial y^{2}}(y, t)\right)^{2} d y
\end{aligned}
$$

Substituting the above expressions in (3.5) we obtain:

$$
\begin{aligned}
& \frac{1}{2} \frac{d}{d t}\left\{\left\|v_{\mu}^{\prime}(t)\right\|^{2}+\int_{0}^{1} \frac{1}{\gamma^{2}}\left[M\left(y, t, \frac{1}{\gamma^{2}}\left\|v_{\mu-1}(t)\right\|^{2}\right)-\frac{m_{0}}{16}\right]\left(\frac{\partial^{2} v_{\mu}}{\partial y^{2}}(y, t)\right)^{2} d y+\right. \\
& \left.+\int_{0}^{1} a(y, t)\left(\frac{\partial^{2} v_{\mu}}{\partial y^{2}}(y, t)\right)^{2} d y\right\}+ \\
& +\frac{\beta^{\prime}}{\gamma}\left(\frac{\partial v_{\mu}^{\prime}}{\partial y}(1, t)\right)^{2}-\frac{\alpha^{\prime}}{\gamma}\left(\frac{\partial v_{\mu}^{\prime}}{\partial y}(0, t)\right)^{2}+ \\
& +\int_{0}^{1} \frac{\gamma^{\prime}}{\gamma^{3}}\left[M\left(y, t, \frac{1}{\gamma^{2}}\left\|v_{\mu-1}(t)\right\|^{2}\right)-\frac{m_{0}}{16}\right]\left(\frac{\partial^{2} v_{\mu}}{\partial y^{2}}(y, t)\right)^{2} d y= \\
& =\left(\left(f_{\mu}(t), v_{\mu}^{\prime}(t)\right)\right)+\frac{1}{2} \int_{0}^{1} \frac{1}{\gamma^{2}} \frac{\partial M}{\partial t}\left(y, t, \frac{1}{\gamma^{2}}\left\|v_{\mu-1}(t)\right\|^{2}\right)\left(\frac{\partial^{2} v_{\mu}}{\partial y^{2}}(y, t)\right)^{2} d y+
\end{aligned}
$$




$$
\begin{aligned}
& +\int_{0}^{1} \frac{1}{\gamma^{2}} \frac{\partial M}{\partial \lambda}\left(y, t, \frac{1}{\gamma^{2}}\left\|v_{\mu-1}(t)\right\|^{2}\right)\left[-\frac{\gamma^{\prime}}{\gamma^{3}}\left\|v_{\mu-1}(t)\right\|^{2}+\right. \\
& \left.+\frac{1}{\gamma^{2}}\left(\left(v_{\mu-1}(t), v_{\mu-1}^{\prime}(t)\right)\right)\right]\left(\frac{\partial^{2} v_{\mu}}{\partial y^{2}}(y, t)\right)^{2} d y+\frac{\gamma^{\prime}}{\gamma}\left\|v_{\mu}^{\prime}(t)\right\|^{2}+ \\
& +\left(\frac{2 \beta^{\prime} \gamma^{\prime}-\beta^{\prime \prime} \gamma}{\gamma^{2}}\right) \frac{\partial v_{\mu}}{\partial y}(1, t) \frac{\partial v_{\mu}^{\prime}}{\partial y}(1, t)+ \\
& +\left(\frac{\alpha^{\prime \prime} \gamma-2 \alpha^{\prime} \gamma^{\prime}}{\gamma^{2}}\right) \frac{\partial v_{\mu}}{\partial y}(0, t) \frac{\partial v_{\mu}^{\prime}}{\partial y}(0, t)+ \\
& +\int_{0}^{1}\left(\frac{\left(\alpha^{\prime \prime}+\gamma^{\prime \prime} y\right) \gamma-2\left(\alpha^{\prime}+\gamma^{\prime} y\right) \gamma^{\prime}}{\gamma^{2}}\right) \frac{\partial^{2} v_{\mu}}{\partial y^{2}}(y, t) \frac{\partial v_{\mu}^{\prime}}{\partial y}(y, t) d y+ \\
& +\int_{0}^{1}\left(\frac{\gamma^{\prime \prime} \gamma-2\left(\gamma^{\prime}\right)^{2}}{\gamma^{2}}\right) \frac{\partial v_{\mu}}{\partial y}(y, t) \frac{\partial v_{\mu}^{\prime}}{\partial y}(y, t) d y-\frac{1}{2} \int_{0}^{1} a^{\prime}(y, t)\left(\frac{\partial^{2} v_{\mu}}{\partial y^{2}}(y, t)\right)^{2} d y .
\end{aligned}
$$

We need modify certains terms in the right hand side of (3.6) in order to obtain a convenient differential inequality.

$$
\text { - } \frac{\partial v_{\mu}}{\partial y}(1, t)=\int_{0}^{1} \frac{\partial}{\partial y}\left(y \frac{\partial v_{\mu}}{\partial y}(y, t)\right) d y \text {. }
$$

Taking the derivatives under the integral, considering the absolute value of both sides, by Cauchy-Schwarz inequality, we obtain

$$
\left|\frac{\partial v_{\mu}}{\partial y}(1, t)\right|_{\mathbb{R}} \leq\left(\int_{0}^{1}\left(\frac{\partial v_{\mu}}{\partial y}(y, t)\right)^{2} d y\right)^{1 / 2}+\left(\int_{0}^{1}\left(\frac{\partial^{2} v_{\mu}}{\partial y^{2}}(y, t)\right)^{2}\right)^{1 / 2}
$$

By Poincaré inequality, since $\lambda_{1}=\pi^{2}$, we obtain

$$
\left|\frac{\partial v_{\mu}}{\partial y}(1, t)\right|_{\mathbb{R}} \leq\left(\frac{1+\pi}{\pi}\right)\left|\frac{\partial^{2} v_{\mu}}{\partial y^{2}}\right|_{L^{2}(0,1)}
$$

- By the same argument we obtain

$$
\left|\frac{\partial v_{\mu}}{\partial y}(0, t)\right|_{\mathbb{R}} \leq\left|\int_{0}^{1} \frac{\partial}{\partial y}\left[(1-y) \frac{\partial v_{\mu}}{\partial y}(y, t)\right] d y\right|_{\mathbb{R}}
$$

or

$$
\left|\frac{\partial v_{\mu}}{\partial y}(0, t)\right|_{\mathbb{R}} \leq\left(\frac{1+\pi}{\pi}\right)\left|\frac{\partial^{2} v_{\mu}}{\partial y^{2}}(t)\right|_{L^{2}(0,1)}
$$




$$
\begin{aligned}
& \text { - }\left(\frac{2 \beta^{\prime} \gamma^{\prime}-\beta^{\prime \prime} \gamma}{\gamma^{2}}\right) \frac{\partial v_{\mu}}{\partial y}(1, t) \frac{\partial v_{\mu}^{\prime}}{\partial y}(1, t) \leq \\
& \leq \frac{2 \beta^{\prime} \gamma^{\prime}}{\gamma^{2}}\left|\frac{\partial v_{\mu}}{\partial y}(1, t)\right|\left|\frac{\partial v_{\mu}^{\prime}}{\partial y}(1, t)\right|+\left(\frac{\beta^{\prime}}{\gamma}\right)^{2}\left|\frac{\partial v_{\mu}}{\partial y}(1, t)\right|\left|\frac{\partial v_{\mu}^{\prime}}{\partial y}(1, t)\right| \leq \\
& \leq\left(\frac{4 \beta^{\prime}\left(\gamma^{\prime}\right)^{2}+\left(\beta^{\prime}\right)^{3}}{2 \gamma^{3}}\right)\left|\frac{\partial v_{\mu}(1, t)}{\partial y}\right|^{2}+\frac{\beta^{\prime}}{\gamma}\left|\frac{\partial v_{\mu}^{\prime}}{\partial y}(1, t)\right|^{2} \text {. } \\
& \text { - }\left(\frac{\alpha^{\prime \prime} \gamma-\alpha^{\prime} \gamma^{\prime}}{\gamma^{2}}\right) \frac{\partial v_{\mu}}{\partial y}(0, t) \frac{\partial v_{\mu}^{\prime}(0, t)}{\partial y}(0, t) \leq \\
& \leq\left(\frac{4\left(-\alpha^{\prime}\right)\left(\gamma^{\prime}\right)^{2}+\left(-\alpha^{\prime}\right)^{3}}{2 \gamma^{3}}\right)\left|\frac{\partial v_{\mu}}{\partial y}(0, t)\right|^{2}-\frac{\alpha^{\prime}}{\gamma}\left|\frac{\partial v_{\mu}^{\prime}}{\partial y}(0, t)\right| \\
& \text { - } \int_{0}^{1} \frac{1}{\gamma^{2}} \frac{\partial M}{\partial \lambda}\left(y, t, \frac{1}{\gamma^{2}}\left\|v_{\mu-1}(t)\right\|^{2}\right)\left[\frac{1}{\gamma^{2}}\left(\left(v_{\mu-1}(t), v_{\mu-1}^{\prime}(t)\right)\right)-\right. \\
& \left.-\frac{\gamma^{\prime}}{\gamma^{3}}\left\|v_{\mu-1}(t)\right\|^{2}\right]\left(\frac{\partial^{2} v_{\mu}}{\partial y^{2}}(y, t)\right)^{2} d y \leq \\
& \leq \int_{0}^{1}\left|\frac{\partial M}{\partial \lambda}\left(y, t, \frac{1}{\gamma^{2}}\left\|v_{\mu-1}(t)\right\|^{2}\right)\right|\left[\frac{\gamma^{\prime}}{\gamma^{5}}\left\|v_{\mu-1}(t)\right\|^{2}+\right. \\
& \left.+\frac{1}{\gamma^{4}}\left\|v_{\mu-1}(t)\right\| \cdot\left\|v_{\mu-1}^{\prime}(t)\right\|\right]\left(\frac{\partial^{2} v_{\mu}}{\partial y^{2}}(y, t)\right)^{2} d y \leq \\
& \leq \frac{1}{2} \int_{0}^{1}\left|\frac{\partial M}{\partial \lambda}\left(y, t, \frac{1}{\gamma^{2}}\left\|v_{\mu-1}(t)\right\|^{2}\right)\right|\left[\left(\frac{1+\gamma^{\prime}}{\pi^{2} \gamma^{5}}\right)\left|\frac{\partial^{2} v_{\mu-1}(t)}{\partial y^{2}}\right|^{2}+\right. \\
& \left.+\frac{1}{\gamma^{3}}\left\|v_{\mu-1}^{\prime}(t)\right\|^{2}\right]\left(\frac{\partial^{2} v_{\mu}}{\partial y^{2}}(y, t)\right)^{2} d y \leq \\
& \leq \frac{1}{2} \int_{0}^{1}\left|\frac{\partial M}{\partial \lambda}\left(y, t, \frac{1}{\gamma^{2}}\left\|v_{\mu-1}(t)\right\|^{2}\right)\right|\left[\frac{4+\sqrt{m_{0}}}{4 \pi^{2} \gamma^{5}}\left|\frac{\partial^{2} v_{\mu-1}(t)}{\partial y^{2}}\right|^{2}+\right. \\
& \left.+\frac{1}{\gamma^{3}}\left\|v_{\mu-1}^{\prime}(t)\right\|^{2}\right]\left(\frac{\partial^{2} v_{\mu}}{\partial y^{2}}(y, t)\right)^{2} d y \text {. }
\end{aligned}
$$

Note that in this last step of the estimate, we employed the hypotheses $\delta \leq \frac{3 \sqrt{m_{0}}}{\gamma_{0}}$ and $\gamma^{\prime} \leq \frac{\delta}{12} \gamma_{0} \leq \frac{\sqrt{m_{0}}}{4}$

- $\int_{0}^{1} \frac{\gamma^{\prime}}{\gamma^{3}}\left[M\left(y, t, \frac{1}{\gamma^{2}}\left\|v_{\mu-1}(t)\right\|^{2}\right)-\frac{m_{0}}{16}\right]\left(\frac{\partial^{2} v_{\mu}}{\partial y^{2}}(y, t)\right)^{2} d y \geq 0$ 
- $\frac{\beta^{\prime}}{\gamma}\left(\frac{\partial v^{\prime}}{\partial y}(1, t)\right)^{2}-\frac{\alpha^{\prime}}{\gamma}\left(\frac{\partial v_{\mu}^{\prime}}{\partial y}(0, t)\right)^{2} \geq 0$

Thus, from the inequalities obtained above, we modify (3.6) giving

$$
\begin{aligned}
& \frac{1}{2} \frac{d}{d t}\left\{\left\|v_{\mu}^{\prime}(t)\right\|^{2}+\int_{0}^{1} \frac{1}{\gamma^{2}}\left[M\left(y, t, \frac{1}{\gamma^{2}}\left\|v_{\mu-1}(t)\right\|^{2}\right)-\left(\alpha^{\prime}+\gamma^{\prime} y\right)^{2}\right]\left|\frac{\partial^{2} v_{\mu}}{\partial y^{2}}(t)\right|^{2} d y\right\} \leq \\
& \left.\leq \frac{1}{\delta}\left\|f_{\mu}(t)\right\|^{2}+\frac{\delta}{4}\left\|v_{\mu}^{\prime}(t)\right\|^{2}+\frac{1}{2} \int_{0}^{1} \frac{1}{\gamma^{2}} \mid \frac{\partial M}{\partial t}\left(y, t, \frac{1}{\gamma^{2}}\left\|v_{\mu-1}(t)\right\|^{2}\right)\right]\left(\frac{\partial^{2} v_{\mu}}{\partial y^{2}}(y, t)\right)^{2} d y+ \\
& +\frac{1}{2} \int_{0}^{1}\left|\frac{\partial M}{\partial \lambda}\left(y, t, \frac{1}{\gamma^{2}}\left\|v_{\mu-1}(t)\right\|^{2}\right)\right|\left[\left(\frac{4+\sqrt{m_{0}}}{4 \pi^{2} \gamma^{5}}\right)\left|\frac{\partial^{2} v_{\mu-1}}{\partial y^{2}}(t)\right|^{2}+\right. \\
& \left.+\frac{1}{\gamma^{3}}\left\|v_{\mu-1}^{\prime}(t)\right\|^{2}\right]\left(\frac{\partial^{2} v_{\mu}}{\partial y^{2}}(y, t)\right)^{2} d y+\frac{\gamma^{\prime}}{\gamma}\left\|v_{\mu}^{\prime}(t)\right\|^{2}+ \\
& +\left(\frac{4 \beta^{\prime}\left(\gamma^{\prime}\right)^{2}+\left(\beta^{\prime}\right)^{3}+4\left(-\alpha^{\prime}\right)\left(\gamma^{\prime}\right)^{2}+\left(-\alpha^{\prime}\right)^{3}}{2 \gamma^{3}}\right)\left(\frac{1+\pi}{\pi}\right)^{2}\left|\frac{\partial^{2} v_{\mu}}{\partial y^{2}}\right|^{2}+ \\
& +\frac{1}{2} \int_{0}^{1} a^{\prime}(y, t)\left(\frac{\partial^{2} v_{\mu}}{\partial y^{2}}(y, t)\right)^{2} d y+ \\
& +\int_{0}^{1}\left|\frac{\gamma^{\prime \prime} \gamma-2\left(\gamma^{\prime}\right)^{2}}{\gamma^{2}}\right|\left|\frac{\partial v_{\mu}}{\partial y}(y, t)\right|\left|\frac{\partial v_{\mu}^{\prime}}{\partial y}(y, t)\right| d y+ \\
& +\int_{0}^{1}\left[\frac{\left.\mid \alpha^{\prime \prime}+\gamma^{\prime \prime} y\right) \gamma|+2| \alpha^{\prime}+\gamma^{\prime} y \mid \gamma^{\prime}}{\gamma^{2}}\right]\left|\frac{\partial^{2} v_{\mu}}{\partial y^{2}}(y, t)\right|\left|\frac{\partial v_{\mu}^{\prime}}{\partial y}(y, t)\right| d y
\end{aligned}
$$

Remark 3.2. From hypothesis (H3) we obtain $\left|\alpha^{\prime \prime}(t)\right| \leq \frac{\left(\alpha^{\prime}\right)^{2}}{\gamma}, \quad\left|\beta^{\prime \prime}\right| \leq \frac{\left(\beta^{\prime}\right)^{2}}{\gamma}$. By definition of $\gamma$ and by (H1) we get $\beta^{\prime} \leq \gamma^{\prime}$ and $\left|\alpha^{\prime}\right| \leq \gamma^{\prime}$.

We obtain

$$
\begin{gathered}
-\frac{1}{2 \gamma^{3}}\left[4 \beta^{\prime}\left(\gamma^{\prime}\right)^{2}+\left(\beta^{\prime}\right)^{3}+4\left(-\alpha^{\prime}\right)\left(\gamma^{\prime}\right)^{2}+\left(-\alpha^{\prime}\right)^{3}\right]= \\
=\frac{1}{2 \gamma^{3}}\left[4 \gamma^{\prime 2}\left(\beta^{\prime}-\alpha^{\prime}\right)+\left(\beta^{\prime}\right)^{3}-\left(\alpha^{\prime}\right)^{3}\right] \leq 3\left(\frac{\gamma^{\prime}}{\gamma}\right)^{3} . \\
\text { - } \frac{1}{\gamma^{2}}\left[\left|\alpha^{\prime \prime}+\gamma^{\prime \prime} y\right| \gamma+2\left|\alpha^{\prime}+\gamma^{\prime} y\right| \gamma^{\prime}\right] \leq 3\left(\frac{\gamma^{\prime}}{\gamma}\right)^{2},
\end{gathered}
$$


since $\left|\alpha^{\prime \prime}+\gamma^{\prime \prime} y\right| \gamma \leq\left|\alpha^{\prime}+\gamma^{\prime} y\right|^{2}<\left(\gamma^{\prime}\right)^{2}$.

$$
\begin{aligned}
& -\quad\left|\frac{\gamma^{\prime \prime} \gamma-2\left(\gamma^{\prime}\right)^{2}}{\gamma^{2}}\right| \leq \frac{\left|\beta^{\prime \prime}\right|+\left|\alpha^{\prime \prime}\right|}{\gamma}+2\left(\frac{\gamma^{\prime}}{\gamma}\right)^{2} \leq 4\left(\frac{\gamma^{\prime}}{\gamma}\right)^{2} \\
& \text { - } \quad \frac{1}{2} a^{\prime}(y, t)=\frac{1}{\gamma^{3}}\left[\gamma^{\prime}\left(\alpha^{\prime}+\gamma^{\prime} y\right)^{2}-\left(\alpha^{\prime \prime}+\gamma^{\prime \prime} y\right)\left(\alpha^{\prime}+\gamma^{\prime} y\right) \gamma\right]- \\
& -\frac{m_{0} \gamma^{\prime}}{16 \gamma^{3}} \leq \frac{1}{\gamma^{3}}\left[\left(\alpha^{\prime}+\gamma^{\prime} y\right)^{2} \gamma^{\prime}+\left|\alpha^{\prime}+\gamma^{\prime} y\right|^{3}\right] \leq 2\left(\frac{\gamma^{\prime}}{\gamma}\right)^{3} .
\end{aligned}
$$

Set $\frac{\partial^{2} v_{\mu}}{\partial y^{2}}$ equal to $\Delta v_{\mu}$. Then $\left|\frac{\partial^{2} v_{\mu}}{\partial y^{2}}(t)\right|_{L^{2}(0,1)}=\left|\Delta v_{\mu}(t)\right|_{L^{2}(0,1)}$ which we write $\left|\Delta v_{\mu}(t)\right|$. Thus, define

$$
z_{\mu}(t)=\frac{1}{2}\left\|v_{\mu}^{\prime}(t)\right\|^{2}+\frac{1}{2 \gamma^{2}} \int_{0}^{1}\left[M\left(y, t, \frac{1}{\gamma^{2}}\left\|v_{\mu-1}(t)\right\|^{2}\right)-\left(\alpha^{\prime}+\gamma^{\prime} y\right)^{2}\right]\left|\Delta v_{\mu}(t)\right|^{2} d y
$$

for all $\mu \in \mathbb{N}$ and $t \in\left[0, T_{\mu}\right]$.

Thus, from (3.7) and the estimates of the coefficients by a power of $\left(\frac{\gamma^{\prime}}{\gamma}\right)$ obtained above, we obtain

$$
\begin{aligned}
& z_{\mu}^{\prime}(t) \leq \frac{1}{\delta}\left\|f_{\mu}(t)\right\|^{2}+\frac{\delta}{4}\left\|v_{\mu}^{\prime}(t)\right\|^{2}+\frac{1}{2 \gamma^{2}} \int_{0}^{1}\left|\frac{\partial M}{\partial t}\left(y, t, \frac{1}{\gamma^{2}}\left\|v_{\mu-1}(t)\right\|^{2}\right)\right|\left|\Delta v_{\mu}(t)\right|^{2} d y+ \\
& +\frac{1}{2} \int_{0}^{1}\left|\frac{\partial M}{\partial \lambda}\left(y, t, \frac{1}{\gamma^{2}}\left\|v_{\mu-1}(t)\right\|^{2}\right)\right|\left[\frac{4+\sqrt{m_{0}}}{4 \pi^{2} \gamma^{5}}\left|\Delta v_{\mu-1}(t)\right|^{2}+\right. \\
& \left.+\frac{1}{\gamma^{3}}\left\|v_{\mu-1}^{\prime}(t)\right\|^{2}\right]\left(\Delta v_{\mu}(y, t)\right)^{2} d y+\frac{\gamma^{\prime}}{\gamma}\left\|v_{\mu}^{\prime}(t)\right\|^{2}+ \\
& +\left[\frac{3(1+\pi)^{2}}{\pi^{2}}+2\right]\left(\frac{\gamma^{\prime}}{\gamma}\right)^{3}\left|\Delta v_{\mu}(t)\right|^{2}+ \\
& +\left(\frac{3 \pi+4}{\pi}\right)\left(\frac{\gamma^{\prime}}{\gamma}\right)^{2}\left|\Delta v_{\mu}(t)\right| \cdot\left\|v_{\mu}^{\prime}(t)\right\| .
\end{aligned}
$$

Observe that in the computation to obtain (3.8) we employed the estimate

$$
4\left(\frac{\gamma^{\prime}}{\gamma}\right)^{2}\left\|v_{\mu}(t)\right\| \cdot\left\|v_{\mu}^{\prime}(t)\right\| \leq \frac{4}{\pi}\left(\frac{\gamma^{\prime}}{\gamma}\right)^{2}\left|\Delta v_{\mu}(t)\right| \cdot\left\|v_{\mu}^{\prime}(t)\right\|
$$

Lemma 3.1. For all $\mu \in \mathbb{N}$, set $\alpha_{\mu}=\operatorname{Ess} S u p\left\{z_{\mu}(t) ; t \in\left[0, T_{\mu}\right]\right\}, \quad \alpha_{\mu}^{\prime}=\frac{32}{15 \pi^{2} m_{0}} \alpha_{\mu}$, $\theta_{\mu}=\left\|\frac{\partial M}{\partial t}\right\|_{L^{\infty}\left(Q \times\left(0, \alpha_{\mu}^{\prime}\right)\right)}$ and $\beta_{\mu}=\left\|\frac{\partial M}{\partial \lambda}\right\|_{L^{\infty}\left(Q \times\left(0, \alpha_{\mu}^{\prime}\right)\right)}$. Then $\alpha_{\mu}$ is finite, $T_{\mu}=T$ 
and

$$
z_{\mu}(t) \leq\left[z_{\mu}(0)+\frac{1}{\delta} \int_{0}^{T}\left\|f_{\mu}(t)\right\|^{2} d t\right] e^{\left(\delta+K_{0} \theta_{\mu-1}+K_{1} \alpha_{\mu-1} \beta_{\mu-1}\right) t} .
$$

for $\mu=1,2, \ldots$.

Proof. It will be done by induction on $\mu \in \mathbb{N}$. First of all we will express the terms in the right hand side of (3.8) in function of $z_{\mu}(t)$.

- Note that from the hypothesis (H2) we have $\gamma^{\prime}<\frac{\delta}{12} \gamma_{0}$ what implies $\left|\alpha^{\prime}+\gamma^{\prime} y\right|<\frac{\sqrt{m_{0}}}{4}$. Thus, by $(\widehat{M} 3)$,

$$
M(y, t, \lambda)-\left(\alpha^{\prime}+\gamma^{\prime} y\right)^{2} \geq M(y, t, \lambda)-\frac{m_{0}}{16}>\frac{15 m_{0}}{16},
$$

what implies

$$
\frac{1}{2 \gamma^{2}} \int_{0}^{1}\left(M\left(y, t, \frac{1}{\gamma^{2}}\left\|v_{\mu-1}(t)\right\|^{2}\right)-\left(\alpha^{\prime}+\gamma^{\prime} y\right)^{2}\right)\left(\Delta v_{\mu}(y, t)\right)^{2} d y \geq \frac{15 m_{0}}{32 \gamma^{2}}\left|\Delta v_{\mu}(t)\right|^{2} .
$$

By the definition of $z_{\mu}(t)$ and the last estimate we get

$$
\text { - }\left|\Delta v_{\mu}(t)\right|^{2} \leq \frac{32 \gamma^{2}}{15 m_{0}} z_{\mu}(t) .
$$

By Poincaré inequality we get

$$
\begin{aligned}
& \frac{1}{\gamma^{2}}\left\|v_{\mu}(t)\right\|^{2} \leq \frac{1}{\gamma^{2}} \frac{1}{\pi^{2}}\left|\Delta v_{\mu}(t)\right|^{2} \leq \\
& \leq \frac{32}{15 m_{0} \pi^{2}} \underset{0 \leq t \leq T_{\mu}}{\operatorname{Ess}} \operatorname{Sup}_{0} z_{\mu}(t)=\frac{32}{15 m_{0} \pi^{2}} \alpha_{\mu}=\alpha_{\mu}^{\prime} .
\end{aligned}
$$

Thus

$$
\text { - } \quad \frac{1}{\gamma^{2}}\left\|v_{\mu}(t)\right\|^{2} \leq \alpha_{\mu}^{\prime}
$$

By $\gamma^{\prime}(t) \leq \frac{\sqrt{m_{0}}}{4}$ and $(3.10)$, we get

$$
\text { - }\left(\frac{\gamma^{\prime}}{\gamma}\right)^{3}\left|\Delta v_{\mu}(t)\right|^{2} \leq \frac{2 \gamma^{\prime}}{15 \gamma} z_{\mu}(t)
$$

From (3.10) and the definition of $z_{\mu}(t)$, we obtain

$$
\text { - }\left(\frac{\gamma^{\prime}}{\gamma}\right)^{2}\left|\Delta v_{\mu}(t)\right||| v_{\mu}^{\prime}(t) \| \leq \frac{2}{\sqrt{15}}\left(\frac{\gamma^{\prime}}{\gamma}\right) z_{\mu}(t)
$$


Now we start the proof of Lemma 3.1. In fact, for $\mu=1$, since $v_{0}(t)=0$ on $[0, T]$, by definition, we obtain

$$
\begin{aligned}
& z_{1}^{\prime}(t) \leq \frac{1}{\delta}\left\|f_{1}(t)\right\|^{2}+\frac{\delta}{4}\left\|v_{1}^{\prime}(t)\right\|^{2}+\frac{1}{2 \gamma^{2}} \int_{0}^{1}\left|\frac{\partial M}{\partial t}(y, t, 0)\right|\left(\Delta v_{1}(y, t)\right)^{2} d y+ \\
& +\frac{\gamma^{\prime}}{\gamma}\left\|v_{1}^{\prime}(t)\right\|^{2}+\left[3\left(\frac{1+\pi}{\pi}\right)^{2}+2\right]\left(\frac{\gamma^{\prime}}{\gamma}\right)^{3}\left|\Delta v_{1}(t)\right|^{2}+ \\
& +\left(\frac{4+3 \pi}{\pi}\right)\left(\frac{\gamma^{\prime}}{\gamma}\right)^{2}\left|\Delta v_{1}(t)\right| \cdot\left\|v_{1}^{\prime}(t)\right\| .
\end{aligned}
$$

Thus, by (3.10)-(3.13) and the notation of the constants fixed in (2.5) we obtain from (3.14)

$$
\begin{gathered}
z_{1}^{\prime}(t) \leq \frac{1}{\delta}\left\|f_{1}(t)\right\|^{2}+\frac{\delta}{2} z_{1}(t)+\theta_{0} K_{0} z_{1}(t)+ \\
+\left(\frac{\gamma^{\prime}}{\gamma}\right) \quad\left\{\frac{2}{15}\left[3\left(\frac{1+\pi}{\pi}\right)^{2}+2\right]+\frac{2}{\sqrt{15}}\left(3+\frac{4}{\pi}\right)\right\} z_{1}(t) \leq \\
\leq \frac{1}{\delta}\left\|f_{1}(t)\right\|^{2}+\left(\frac{\delta}{2}+\theta_{0} K_{0}+\frac{6 \gamma^{\prime}}{\gamma}\right) z_{1}(t) .
\end{gathered}
$$

But $\frac{6 \gamma^{\prime}}{\gamma} \leq 6 \frac{\gamma^{\prime}}{\gamma_{0}} \leq \frac{\delta}{2}$ then

$$
z_{1}^{\prime}(t) \leq \frac{1}{\delta}\left\|f_{1}(t)\right\|+\left(\delta+\theta_{0} K_{0}\right) z_{1}(t)
$$

or

$$
z_{1}(t) \leq\left(z_{1}(0)+\frac{1}{\delta} \int_{0}^{T}\left\|f_{1}(t)\right\|^{2} d t\right) e^{\left(\delta+K_{0} \theta_{0}\right) t}
$$

and (3.9) is true for $\mu=1$. Furthermore, by (3.15)

$$
\left\|v_{1}^{\prime}(t)\right\|^{2}+\left|\Delta v_{1}(t)\right|^{2}<C_{1}
$$

for all $0 \leq t \leq T_{1}$, and $v_{1}$ is extended to $[0, T]$ as a solution of the approximate scheme (3.1).

To complete the inductive process we assume it is true for $\mu-1$, that is, $\alpha_{\mu-1}$ is finite, $T_{\mu-1}=T$ and (3.9) is true for $\mu-1$ and $t \in[0, T]$.

To begin we modify (3.8) writting its right hand side in function of $z_{\mu}(t)$ by means of (3.10)-(3.13). In fact, note that

$$
\begin{aligned}
- & \frac{1}{2 \gamma^{2}} \int_{0}^{1}\left|\frac{\partial M}{\partial t}\left(y, t, \frac{1}{\gamma^{2}}\left\|v_{\mu-1}(t)\right\|^{2}\right)\right|\left(\Delta v_{\mu}(y, t)\right)^{2} d y \leq \frac{1}{2 \gamma^{2}} \theta_{\mu-1}\left|\Delta v_{\mu}(t)\right|^{2} \leq \\
\leq & \frac{16}{15 m_{0}} \theta_{\mu-1} z_{\mu}(t) .
\end{aligned}
$$


- $\frac{1}{2} \int_{0}^{1}\left|\frac{\partial M}{\partial \lambda}\left(y, t, \frac{1}{\gamma^{2}}\left\|v_{\mu-1}(t)\right\|^{2}\right)\right|\left[\frac{4+\sqrt{m_{0}}}{4 \pi^{2} \gamma^{5}}\left|\Delta v_{\mu-1}(t)\right|^{2}+\right.$ $\left.+\frac{1}{\gamma^{3}}\left\|v_{\mu-1}^{\prime}(t)\right\|^{2}\right]\left(\Delta v_{\mu}(y, t)\right)^{2} d y \leq$

$$
\begin{aligned}
& \leq \frac{1}{2} \beta_{\mu-1} \int_{0}^{1}\left[\frac{4+\sqrt{m_{0}}}{4 \pi^{2} \gamma^{5}} \frac{32 \gamma^{2}}{15 m_{0}} \alpha_{\mu-1}+\frac{2}{\gamma^{3}} \alpha_{\mu-1}\right]\left(\Delta v_{\mu}(y, t)\right)^{2} d y= \\
& =\beta_{\mu-1} \alpha_{\mu-1}\left[\frac{16+4 \sqrt{m_{0}}}{15 m_{0} \pi^{2}}+1\right] \frac{32}{15 m_{0} \gamma_{0}} z_{\mu}(t)
\end{aligned}
$$

- $\frac{\gamma^{\prime}}{\gamma}\left\|v_{\mu}^{\prime}(t)\right\|^{2} \leq 2 \frac{\gamma^{\prime}}{\gamma} z_{\mu}(t)$

- $\left[3\left(\frac{1+\pi}{\pi}\right)^{2}+2\right]\left(\frac{\gamma^{\prime}}{\gamma}\right)^{3}\left|\Delta v_{\mu}(t)\right|^{2} \leq$ $\leq\left[3\left(\frac{1+\pi}{\pi}\right)^{2}+2\right] \frac{2 \gamma^{\prime}}{15 \gamma} z_{\mu}(t)=$

$$
=\left[\frac{2}{5}\left(\frac{1+\pi}{\pi}\right)^{2}+\frac{4}{15}\right]\left(\frac{\gamma^{\prime}}{\gamma}\right) z_{\mu}(t)
$$

- $\left(\frac{4+3 \pi}{\pi}\right)\left(\frac{\gamma^{\prime}}{\gamma}\right)^{2}\left|\Delta v_{\mu}(t)\right|\left\|v_{\mu}^{\prime}(t)\right\| \leq$

$$
\leq\left(\frac{3 \pi+4}{\pi}\right) \frac{2 \gamma^{\prime}}{\sqrt{15} \gamma} z_{\mu}(t)
$$

Substituting (3.14)-(3.18) in (3.7) we obtain

$$
\begin{aligned}
& z_{\mu}^{\prime}(t) \leq \frac{1}{\delta}\left\|f_{\mu}(t)\right\|^{2}+\left[\frac{\delta}{2}+\frac{16}{15 m_{0}} \theta_{\mu-1}+\right. \\
& +\frac{32}{15 m_{0} \gamma_{0}}\left(\frac{16+4 \sqrt{m_{0}}}{15 \pi^{2} m_{0}}+1\right) \beta_{\mu-1} \alpha_{\mu-1}+ \\
& \left.+\frac{\gamma^{\prime}}{\gamma}\left\{\frac{2}{5}\left(\frac{1+\pi}{\pi}\right)^{2}+\frac{2}{\sqrt{15}}\left(3+\frac{4}{\pi}\right)+\frac{4}{15}\right\}\right] z_{\mu}(t) \leq \\
& \leq \frac{1}{\delta}\left\|f_{\mu}(t)\right\|^{2}+\left[\frac{\delta}{2}+K_{0} \theta_{\mu-1}+K_{1} \beta_{\mu-1} \alpha_{\mu-1}+\frac{6 \gamma^{\prime}}{\gamma}\right] z_{\mu}(t)
\end{aligned}
$$

or

$$
z_{\mu}^{\prime}(t) \leq \frac{1}{\delta}\left\|f_{\mu}(t)\right\|^{2}+\left(\delta+K_{0} \theta_{\mu-1}+K_{1} \alpha_{\mu-1} \beta_{\mu-1}\right) z_{\mu}(t)
$$

then for $0 \leq t \leq T_{\mu}$, we have

$$
z_{\mu}(t) \leq\left[z_{\mu}(0)+\frac{1}{\delta} \int_{0}^{T}\left\|f_{\mu}(t)\right\|^{2} d t\right] e^{\left(\delta+K_{0} \theta_{\mu-1}+K_{1} \alpha_{\mu-1} \beta_{\mu-1}\right) t}
$$


By hypothesis the right hand side is bounded by constant independent of $\mu$ for all $t \in\left[0, T_{\mu}\right]$. Thus the solution $v_{\mu}$ is extended for all $t \in[0, T]$, and (3.9) is true for all $t \in[0, T]$ and all $\mu \in \mathbb{N}$ what proves Lemma 3.1.

Set

$$
\tau_{\mu}=z_{\mu}(0)+\frac{1}{\delta} \int_{0}^{T}\left\|f_{\mu}(t)\right\|^{2} d t
$$

By (3.2), (3.3) and the definition of $z_{\mu}(t)$, we obtain:

$$
\tau_{\mu} \leq \frac{1}{2}\left[\left\|\varphi_{1}\right\|^{2}+\frac{K_{2}}{\gamma_{0}^{2}}\left|\Delta \varphi_{0}\right|^{2}\right]+\frac{1}{\delta} \int_{0}^{T}\|f(t)\|^{2} d t .
$$

From the condition (2.7) on $\varphi_{0}, \varphi_{1}$ and $f$, we obtain

$$
\tau_{\mu} \leq \frac{\delta^{2}}{2}\left(1+\frac{K_{2}}{\gamma_{0}^{2}}\right)+\delta
$$

or

$$
\tau_{\mu} \leq K_{3} \delta^{2}+\delta=K_{\delta} \text { for all } \mu \in \mathbb{N}
$$

with $K_{3}=\frac{1}{2}\left(1+\frac{K_{2}}{\gamma_{0}^{2}}\right)$.

Lemma 3.2. For all $\mu \in \mathbb{N}$ and $t \in[0, T]$, we have

$$
z_{\mu}(t) \leq 2 C_{0},
$$

with

$$
C_{0}=K_{\delta}\left(1+e^{\left(1+K_{0} \theta_{0}\right) T}\right)
$$

and $K_{\delta}=K_{3} \delta^{2}+\delta$.

Proof. We will do it by induction. For $\mu=1$ we obtain from (3.15) and (3.21),

$$
z_{1}(t) \leq K_{\delta} e^{\left(\delta+K_{0} \theta_{0}\right) T} \leq C_{0} \leq 2 C_{0}
$$

and it is true for $\mu=1$.

Suppose (3.22) true for $\mu$. Then we have

$$
\text { - } \alpha_{\mu} \leq 2 C_{0} ; \quad \alpha_{\mu}^{\prime} \leq K_{4} ; \quad \beta_{\mu} \leq K_{5} \text { and } \theta_{\mu} \leq \frac{\log 2}{3 K_{0} T} .
$$

We have by hypothesis of induction, Lemma 3.2 true for $\mu \in \mathbb{N}$. Thus from (3.21) we have

$$
z_{\mu+1}(t) \leq K_{\delta} e^{\left(\delta+K_{0} \theta_{m}+K_{1} \alpha_{\mu} \beta_{\mu}\right) t} .
$$

Note that $K_{\delta}<C_{0}=K_{\delta}\left(1+e^{\left(1+K_{0} \theta_{0}\right)}\right)$. Thus, we need only prove the estimate

$$
\left(\delta+K_{0} \theta_{\mu}+K_{1} \alpha_{\mu} \beta_{\mu}\right) t \leq \log 2, \quad 0 \leq t \leq T .
$$


In fact, we have by (3.24)

$$
\begin{aligned}
& \left(\delta+K_{0} \theta_{\mu}+K_{1} \alpha_{\mu} \beta_{\mu}\right) \leq\left(\delta+\frac{\log 2}{3 T}+2 K_{1} K_{5} C_{0}\right)= \\
& =\delta+\frac{\log 2}{3 T}+2 K_{1} K_{5}\left\{\left(K_{3} \delta^{2}+\delta\right)\left(1+e^{\left(1+K_{0} \theta_{0}\right) T}\right)\right\}= \\
& =\left(1+2 K_{1} K_{5} \theta\right) \delta+\left(2 K_{1} K_{3} K_{5} \theta\right) \delta^{2}+\frac{\log 2}{3 T} \leq \\
& \leq \frac{\log 2}{3 T}+\frac{\log 2}{3 T}+\frac{\log 2}{3 T}=\frac{\log 2}{T}
\end{aligned}
$$

by the definition of $\delta$ given in (2.5).

Thus we obtain

$$
\left(\delta+K_{0} \theta_{\mu}+K_{1} \alpha_{\mu} \beta_{\mu}\right) t \leq \log 2
$$

or

$$
e^{\left(\delta+K_{0} \theta_{\mu}+K_{1} \alpha_{\mu} \beta_{\mu}\right) t} \leq 2
$$

and

$$
z_{\mu}(t) \leq 2 C_{0} \quad \text { for all } \quad 0 \leq t \leq T
$$

which proves Lemma 3.2.

It follows that there exists a constant $C_{1}>0$ independent $\mu \in \mathbb{N}$ such that

$$
\left\|v_{\mu}^{\prime}(t)\right\|^{2}+\left|\Delta v_{\mu}(t)\right|^{2}<C_{1}, \text { for all } \mu \in \mathbb{N} \text { and all } t \in[0, T]
$$

\section{Estimate (2)}

Set $w=v_{\mu}^{\prime \prime}(t)$ in (3.1) observing Remark 2.2 and the estimate (3.25) we obtain

$$
\left|v_{\mu}^{\prime \prime}(t)\right|^{2} \leq \frac{1}{\gamma_{0}^{2}}\left(K_{6}+\gamma_{0}^{2}+m_{0}\right) \sqrt{C_{1}}|f(t)|^{2}
$$

with

$$
K_{6}=\|M\|_{L^{\infty}}\left(Q \times\left(0, \frac{C_{1}}{\pi^{2} \gamma_{0}^{2}}\right)\right)
$$

Consequently

$$
\int_{0}^{T}\left|v_{\mu}^{\prime \prime}(t)\right|^{2} d t<C_{2} \quad \text { for all } \quad \mu \in \mathbb{N},
$$

what is the Estimate (2).

Now, from the estimates (3.25), (3.26) and Newton-Leibniz theorem of calculus, we obtain

$$
|| v_{\mu}(t)-v_{\mu}(s)|| \leq \sqrt{C_{1}}|t-s|, \quad\left|v_{\mu}^{\prime}(t)-v_{\mu}^{\prime}(s)\right| \leq \sqrt{C_{2}}|t-s|^{1 / 2},
$$


for all $t, s \in[0, T]$.

Thus, by (3.25), (3.26) and the equicontinuity above for $\left(v_{\mu}\right)_{\mu \in \mathbb{N}}$ and $\left(v_{\mu}^{\prime}\right)_{\mu \in \mathbb{N}}$, we obtain, by Ascoli's theorem, vector version, see Royden[25], Lions [15], that there exists a function

$$
v \in C\left([0, T] ; H_{0}^{1}(0,1)\right) \cap C^{1}\left([0, T] ; L^{2}(0,1)\right)
$$

and a subsequence $\left(v_{\mu_{j}}\right)$ of $\left(v_{\mu}\right)$ such that

$$
\begin{gathered}
v_{\mu_{j}} \rightarrow v \quad \text { strongly } H_{0}^{1}(0,1), \text { uniformly on }[0, T] \\
v_{\mu_{j}}^{\prime} \rightarrow v^{\prime} \text { strongly } L^{2}(0,1), \text { uniformly on }[0, T] .
\end{gathered}
$$

These convergences are sufficient to pass to the limits in the linear terms of the approximate scheme. To obtain the limits of the nonlinear term of the approximate scheme we need the proposition we prove below. Observe that the nonlinear term depends of $v_{\mu_{j}-1}$.

Proposition 3.1. We have

$$
\lim _{\mu \rightarrow \infty}\left\|v_{\mu+1}(t)-v_{\mu}(t)\right\|=0, \text { uniformly on }[0, T] \text {. }
$$

Proof. For all $\mu \in \mathbb{N}$ set $v_{\mu+1}(t)-v_{\mu}(t)=w_{\mu}(t)$ and define

$$
\begin{aligned}
& \psi_{\mu}(t)=\frac{1}{2}\left|w_{\mu}^{\prime}(t)\right|^{2}+\frac{1}{2} \int_{0}^{1} \frac{1}{\gamma^{2}}\left[M\left(y, t, \frac{1}{\gamma^{2}} \|\left. v_{\mu-1}(t)\right|^{2}\right)-\right. \\
& \left.-\left(\alpha^{\prime}+\gamma^{\prime} y\right)^{2}\right]\left(\frac{\partial w_{\mu}}{\partial y}(y, t)\right)^{2} d y .
\end{aligned}
$$

By hypothesis $M(y, t, \lambda) \geq m_{0}>0$, then

$\frac{1}{\gamma^{2}} \int_{0}^{1}\left[M\left(y, t, \frac{1}{\gamma^{2}}\left\|v_{\mu-1}(t)\right\|^{2}\right)-\left(\alpha^{\prime}+\gamma^{\prime} y\right)^{2}\right]\left(\frac{\partial w_{\mu}}{\partial y}(y, t)\right)^{2} d y \geq \frac{15 m_{0}}{16 \gamma^{2}(T)}\left\|w_{\mu}(t)\right\|^{2}$,

because $\left(\alpha^{\prime}+\gamma^{\prime} y\right)^{2} \leq \frac{m_{0}}{16}$, by hypothesis (H2).

Thus we obtain

$$
\left\|w_{\mu}(t)\right\|^{2} \leq C_{4} \psi_{\mu}(t) \text { for all } t \in[0, T] \text { and } \mu \in \mathbb{N} .
$$

Then, to prove Proposition 3.1 it is sufficient to prove that

$$
\lim _{\mu \rightarrow \infty} \psi_{\mu}(t)=0 \quad \text { uniformly on } \quad[0, T]
$$


We take the derivative of $\psi$ what gives

$$
\begin{aligned}
& \psi^{\prime}(t)=\int_{0}^{1} \frac{1}{\gamma^{2}}\left[M\left(y, t, \frac{1}{\gamma^{2}}\left\|v_{\mu}(t)\right\|^{2}\right)-\left(\alpha^{\prime}+\gamma^{\prime} y\right)^{2}\right] \frac{\partial w_{\mu}}{\partial y} \frac{\partial w_{\mu}^{\prime}}{\partial y} d y+ \\
& +\frac{1}{2} \int_{0}^{1} \frac{d}{d t}\left[\frac{1}{\gamma^{2}}\left(M\left(y, t, \frac{1}{\gamma^{2}}\left\|v_{\mu}(t)\right\|^{2}\right)-\left(\alpha^{\prime}+\gamma^{\prime} y\right)^{2}\right)\right]\left(\frac{\partial w_{\mu}}{\partial y}(y, t)\right)^{2} d y+ \\
& +\frac{1}{2} \frac{d}{d t}\left|w_{\mu}^{\prime}(t)\right|^{2}
\end{aligned}
$$

Now we obtain $\frac{d}{d t}\left|w_{\mu}^{\prime}(t)\right|^{2}$ by means of the approximate scheme. In fact, we evaluate (3.1) at $\mu+1$ and $\mu$. Then we sum and subtract

$$
\left[M\left(y, t, \frac{1}{\gamma^{2}}\left\|v_{\mu}(t)\right\|^{2}\right)-\frac{m_{0}}{16}\right] \Delta v_{\mu}(y, t)
$$

and observing that $w_{\mu}=v_{\mu+1}-v_{\mu}$, we have, with $w=w_{\mu}^{\prime}(t)$ in (3.1),

$$
\begin{aligned}
& \frac{1}{2} \frac{d}{d t}\left|w_{\mu}^{\prime}(t)\right|^{2}=\int_{0}^{1} \frac{1}{\gamma^{2}}\left[M\left(y, t, \frac{1}{\gamma^{2}}\left\|v_{\mu}(t)\right\|^{2}\right)-\frac{m_{0}}{16}\right] \Delta v_{\mu}(y, t) w_{\mu}^{\prime}(y, t) d y+ \\
& +\int_{0}^{1} \frac{1}{\gamma^{2}}\left[M\left(y, t, \frac{1}{\gamma^{2}}\left\|v_{\mu}(t)\right\|^{2}\right)-M\left(y, t, \frac{1}{\gamma^{2}}\left\|v_{\mu-1}(t)\right\|^{2}\right)\right] \Delta v_{\mu}(y, t) w_{\mu}^{\prime}(y, t) d y+ \\
& +\int_{0}^{1}\left[b(y, t) \frac{\partial w_{\mu}^{\prime}}{\partial y}-\frac{\partial a}{\partial y}(y, t) \frac{\partial w_{\mu}}{\partial y}(y, t)-a(y, t) \Delta w_{\mu}(y, t)\right] w_{\mu}^{\prime}(y, t) d y- \\
& -\int_{0}^{1}\left[c(y, t) \frac{\partial w_{\mu}}{\partial y}(y, t)-f_{\mu+1}(y, t)-f_{\mu}(y, t)\right] w_{\mu}^{\prime}(y, t) d y
\end{aligned}
$$


Substituting $\frac{1}{2} \frac{d}{d t}\left|w_{\mu}^{\prime}(t)\right|^{2}$ given above in (3.28) we obtain

$$
\begin{aligned}
& \left.\psi_{\mu}^{\prime}(t)=\int_{0}^{1} \frac{1}{\gamma^{2}}\left[M\left(y, t, \frac{1}{\gamma^{2}}\left\|v_{\mu}(t)\right\|^{2}\right)-\left(\alpha^{\prime}+\gamma^{\prime} y\right)^{2}\right)\right] \Delta w_{\mu}(y, t) w_{\mu}^{\prime}(y, t) d y+ \\
& +\int_{0}^{1} \frac{1}{\gamma^{2}}\left[M\left(y, t, \frac{1}{\gamma^{2}}\left\|v_{\mu}(t)\right\|^{2}\right)-M\left(y, t, \frac{1}{\gamma^{2}}\left\|v_{\mu-1}(t)\right\|^{2}\right)\right] \Delta v_{\mu}(y, t) w_{\mu}^{\prime}(y, t) d y+ \\
& +\int_{0}^{1} \frac{\partial b}{\partial y}(y, t)\left(w_{\mu}^{\prime}(y, t)\right)^{2} d y+\int_{0}^{1}\left(\frac{\partial a}{\partial y}(y, t)-c(y, t)\right) \frac{\partial v_{\mu}}{\partial y}(y, t) w_{\mu}^{\prime}(y, t) d y+ \\
& +\int_{0}^{1} \frac{1}{\gamma^{2}}\left[M\left(y, t, \frac{1}{\gamma^{2}}\left\|v_{\mu}(t)\right\|^{2}\right)-\left(\alpha^{\prime}+\gamma^{\prime} y\right)^{2}\right] \frac{\partial v_{\mu}}{\partial y}(y, t) \frac{\partial w_{\mu}^{\prime}}{\partial y}(y, t) d y+ \\
& +\frac{1}{2} \int_{0}^{1} \frac{d}{d t}\left\{\frac{1}{\gamma^{2}}\left[M\left(y, t, \frac{1}{\gamma^{2}}\left\|v_{\mu}(t)\right\|^{2}\right)-\left(\alpha^{\prime}+\gamma^{\prime} y\right)^{2}\right]\right\}\left(\frac{\partial v_{\mu}}{\partial y}(y, t)\right)^{2}+ \\
& +\int_{0}^{1}\left(f_{\mu+1}(y, t)-f_{\mu}(y, t)\right) w_{\mu}^{\prime}(y, t) d y .
\end{aligned}
$$

By the estimates obtained for the solution $v_{\mu}(y, t)$, we select a constant $C_{5}>0$, independent of $\mu \in \mathbb{N}$ and $t \in[0, T]$ such that from the expression above for $\psi_{\mu}(t)$ gives

$$
\begin{aligned}
& \psi_{\mu}^{\prime}(t) \leq C_{5}\left[\left\|w_{\mu-1}(t)\right\|^{2}+\left|w_{\mu}^{\prime}(t)\right|^{2}+|| w_{\mu}(t) \|\left.\right|^{2}\right]+ \\
& +\frac{1}{2}\left|f_{\mu+1}(t)-f_{\mu}(t)\right|^{2} \leq C_{5}\left(\psi_{\mu-1}+\psi_{\mu}\right)+\frac{1}{2}\left|f_{\mu+1}(t)-f_{\mu}(t)\right|^{2} .
\end{aligned}
$$

Integrating this differential inequality we get

$$
\psi_{\mu}(t) \leq e^{C_{5} T}\left[\psi_{\mu}(0)+\frac{1}{2} \int_{0}^{T}\left|f_{\mu+1}(t)-f_{\mu}(t)\right|^{2} d t\right]+C_{5} e^{C_{5} T} \int_{0}^{t} \psi_{\mu-1}(s) d s .
$$

Set

$$
\Gamma_{\mu}=\psi_{\mu}(0)+\frac{1}{2} \int_{0}^{T}\left|f_{\mu+1}(t)-f_{\mu}(t)\right|^{2} d t
$$

we obtain

$$
\lim _{\mu \rightarrow \infty} \Gamma_{\mu}=0
$$

Let be

$$
C_{6}=\operatorname{Max}\left\{C_{5} T, C_{5} e^{C_{5} T}, \operatorname{Ess} \operatorname{Sup} \psi_{1}(t)\right\}
$$

what implies

$$
\psi_{1}(t) \leq C_{6} \text { and } \psi_{\mu}(t) \leq C_{6} \Gamma_{\mu}+C_{6} \int_{0}^{t} \psi_{\mu-1}(s) d s, \text { for } \mu=2,3, \ldots
$$


As a consequence of the above recurrence inequality we obtain

$$
\psi_{\mu}(t) \leq C_{6} \sum_{\nu=0}^{\mu-1} \frac{\left(C_{6} t\right)^{\nu}}{\nu !} \Gamma_{\mu-\nu}
$$

for all $t \in[0, T]$ and $\mu=2,3, \ldots$

Note that $\lim _{\mu \rightarrow \infty} \Gamma_{\mu}=0$ and $\sum_{\nu=0}^{\infty} \frac{\left(C_{6} T\right)^{\nu}}{\nu !}=e^{C_{6} T}$.

This implies that

$$
\lim _{\mu \rightarrow \infty} \psi_{\mu}(t)=0 \quad \text { uniformly on }[0, T],
$$

which proves Proposition 3.1.

\section{Convergence of the Nonlinear Term}

From the Proposition 3.1 it follows that

$$
\lim _{\nu \rightarrow \infty}\left\|v_{\mu_{\nu}-1}(t)\right\|^{2}=\|v(t)\|^{2} .
$$

We employ the identity $\left.|| a\right|^{2}-|b|^{2}|\leq C| a-b \mid$ and $w_{\mu_{\nu}}(t)=v_{\mu_{\nu}-1}-v_{\mu_{\nu}}(t)$ is a subsequence of the sequence $w_{\mu}(t)=v_{\mu+1}(t)-v_{\mu}(t)$. Then we employ Proposition 3.1 .

Now consider

$$
\begin{aligned}
& \left|M\left(y, t, \frac{1}{\gamma^{2}}\left\|v_{\mu_{\nu}-1}(t)\right\|^{2}\right)-M\left(y, t, \frac{1}{\gamma^{2}}\|v(t)\|^{2}\right)\right|= \\
& =\left|\int_{\frac{1}{\gamma^{2}}\|v(t)\|^{2}}^{\frac{1}{\gamma^{2}}\left\|v_{\mu_{\nu}-1}(t)\right\|^{2}} \frac{\partial M}{\partial \lambda}(y, t, \lambda) d \lambda\right| \leq C\left|\left\|v_{\mu_{\nu}-1}(t)\right\|^{2}-\|v(t)\|^{2}\right| .
\end{aligned}
$$

Here we employ the notation $\phi(y, t, \lambda)=\phi(t, \lambda)(y)$. Thus

$$
\begin{aligned}
& \left|M\left(t, \frac{1}{\gamma^{2}}\left\|v_{\mu_{\nu}-1}(t)\right\|^{2}\right) \cdot v-M\left(t, \frac{1}{\gamma^{2}}\|v(t)\|^{2}\right) \cdot v\right|_{L^{2}(0,1)}= \\
& =\int_{0}^{1}\left|M\left(y, t, \frac{1}{\gamma^{2}}\left\|v_{\mu_{\nu}-1}(t)\right\|^{2}\right)-M\left(y, t, \frac{1}{\gamma^{2}}\|v(t)\|^{2}\right)\right|^{2} \cdot|v(y)|^{2} d y \leq \\
& \leq C\left|\left\|v_{\mu_{\nu}-1}(t)\right\|^{2}-\|v(t)\|^{2}\right|^{2}|v(t)|^{2} .
\end{aligned}
$$

Then

$$
M\left(t, \frac{1}{\gamma^{2}}\left\|v_{\mu_{\nu}-1}(t)\right\|^{2}\right) \cdot v \rightarrow M\left(t, \frac{1}{\gamma^{2}}\|v(t)\|^{2}\right) \cdot v \text { in } L^{2}(0,1)
$$


It follows that we can pass to the limit in the approximate scheme and conclude that $v$ is a solution claimed in the Theorem 2.1 that is

$$
L v(t)=f(t) \text { in } L^{2}(0,1) \text {, for all } t \in[0, T] .
$$

To complete the proof we need to show the uniqueness.

In fact, let $v, \hat{v}$ be two solutions of Theorem 2.1 and set $w=v-\hat{v}$. Then $w$ is solution of the equation

$$
\begin{aligned}
& w^{\prime \prime}(y, t)-\frac{1}{\gamma^{2}}\left(M\left(y, t, \frac{1}{\gamma^{2}}\|v(t)\|^{2}\right)-\frac{m_{0}}{16}\right) \Delta w(y, t)+ \\
& +\frac{1}{\gamma^{2}}\left[M\left(y, t, \frac{1}{\gamma^{2}}\|\hat{v}(t)\|^{2}\right)-M\left(y, t, \frac{1}{\gamma^{2}}\|v(t)\|^{2}\right)-\frac{m_{0}}{8}\right] \Delta w(y, t)+ \\
& +b(y, t) \frac{\partial w^{\prime}}{\partial y}(y, t)-\frac{\partial a}{\partial y}(y, t) \frac{\partial w}{\partial y}(y, t)-a(y, t) \Delta w(y, t)+ \\
& +c(y, t) \frac{\partial w}{\partial y}(y, t)=0
\end{aligned}
$$

with initial conditions $w(y, 0)=w^{\prime}(y, 0)=0$, for $y \in(0,1)$.

To prove that $w(y, t)=0$ in $Q$ we employ the argument of the proof of the Proposition 3.1. In fact, we consider the function

$$
\psi(t)=\frac{1}{2}\left|w^{\prime}(t)\right|^{2}+\frac{1}{2 \gamma^{2}} \int_{0}^{1}\left[M\left(y, t, \frac{1}{\gamma^{2}}\|v(t)\|^{2}\right)-\left(\alpha^{\prime}+\gamma^{\prime} y\right)^{2}\right]\left(\frac{\partial w}{\partial y}(y, t)\right)^{2} d y
$$

and we prove that

$$
\|w(t)\|^{2} \leq C \psi(t), \quad \text { for all } t \in[0, T] .
$$

Then, to obtain uniqueness, it is sufficient to prove that $\psi(t)=0$ in $[0, T]$. As in the proof of the Proposition 3.1 we obtain a constant $C_{8}>0$ such that

$$
\psi^{\prime}(t) \leq C_{8} \psi(t) \quad \text { for } \quad t \in[0, T]
$$

what implies that $\psi(t)=0$ for all $t \in[0, T]$.

Proof of Theorem 2.2. Suppose $\widehat{M}, u_{0}, u_{1} \hat{f}$ given as in Theorem 2.2. We consider the mapping

$$
\phi: \widehat{Q} \rightarrow Q
$$

such that

$$
\phi(x, t)=(y, t) \quad \text { with } \quad y=\frac{x-\alpha}{\gamma}
$$


We set

$$
\begin{aligned}
& v_{0}(y)=u_{0}\left(\alpha_{0}+\gamma_{0} y\right) \\
& v_{1}(y)=u_{1}\left(\alpha_{0}+\gamma_{0} y\right) \\
& f(y, t)=\hat{f}(\alpha(t)+\gamma(t) y, t)
\end{aligned}
$$

and

$$
M(y, t, \lambda)=\widehat{M}(\alpha(t)+\gamma(t) y, t, \lambda) .
$$

Then, $M(y, t, \lambda), v_{0}, v_{1}, f$ satisfies the condition of Theorem 2.1. Furthermore

$$
\left\|v_{0}\right\|^{2}=\gamma_{0}\left\|u_{0}\right\|_{0}^{2}, \quad\left|\Delta v_{0}\right|^{2}=\gamma_{0}^{3}\left|\Delta u_{0}\right|_{0}^{2}, \quad\left\|v_{1}\right\|^{2}=\gamma_{0}\left\|u_{1}\right\|_{0}^{2}
$$

and $\|f(t)\|^{2}=\gamma(t)\|\hat{f}(t)\|_{t}^{2}$. It follows from (2.12) that we obtain (2.7). Thus, by Theorem 2.1, there exists a unique function $v=v(y, t)$ solution of the problem (2.4). By defining $u(x, t)=v\left(\frac{x-\alpha}{\gamma}, t\right)$ and after some computations we can see that $u=u(x, t)$ is the unique solution of the problem (2.1).

Acknowledgements. We wish to acknowledge the Referee of the "Revista Matemática Complutense", for his constructive remarks and corrections in the manuscript.

\section{References}

[1] A. Arosio \& S. Spagnolo. Global solution to the Cauchy problem for a nonlinear hyperbolic equation. Nonlinear Partial Differential Equations and Their Applications, Collège de France Seminar, Vol. 16, H. Brezis and J.L. Lions edts., Pitman, London, 1984.

[2] D. Bainov \& E. Minchev. Above estimates of the interval of existence of solutions of the nonlinear Kirchhoff equation. Comptes Rendus de l'Academi Bulgare des Sciences, Tome $48 \mathrm{n}^{\mathrm{O}} 5$ (1995) pp. 13-14.

[3] S. Bernstein. Sur une classe d'équations fonctionelles aux derivées partielles. Isv. SSSR Math. 4 (1940) pp. 17-20.

[4] H. Brezis. Analyse Fonctionelles (Théorie and Applications), Masson, Paris 1983.

[5] C.E. Carrier. On the vibrations problem of elastic strings, Q.J. Appl. Math. (1953) pp. 151-165.

[6] R.W. Dickey. Infinity system of semilinear oscilations equations related to the string, Proc. of the A.M.S., vol. 23 (1969) pp. 459-468.

[7] Y. Ebihara, Y. Tanaka and Y. Nakashina. On exact solutions of Kirchhoff quasilinear hyperbolic equations with absorving term. Fukuoka University Science Repports Vol. 22, n 2 (1992) pp. 97-106.

[8] C.L. Frota. Non local solutions of a nonlinear hyperbolic partial differential equation. Port. Math. Vol. 51 Fasc. 3 (1994) pp. 455-473.

[9] M. Hazoya, Y. Yamada. On some nonlinear wave equations II, Global existence and energy decay of solutions. J. Fac. Sci. (Univ. Tokyo) vol. 38, no 2 (1991) pp. 230-250. 
[10] I. Shih-Liu, M. Rincon. Kirchhoff-Carrier string with moving boundary. Numerical Solutions, Preprint IM-UFRJ, 1999.

[11] G. Kirchhoff. Vorlesungen der Mechanik, Taube Leipzig (1883), §7, p. 444.

[12] J. Limaco and L.A. Medeiros. Vibrations of elastic membranes with moving boundaries. Nonlinear Analysis, TMA, 45 (2001) pp. 363-382.

[13] J. Limaco and L.A. Medeiros. Kirchhoff-Carrier elastic strings in noncylindrical domains. Portugaliae Mathematica Vol. 56, Fasc. 4 (1999) pp. 465-500.

[14] J.L. Lions. On Some Questions in Boundary Value Problems of Mathematical Physics. Contemporary Development in Continuum Mechanics and Partial Differential Equations. Edited by G.M. de La Penha and L.A. Medeiros, North-Holland, Amsterdam (1978) pp. $285-346$.

[15] J.L. Lions. Quelques méthodes de résolution des problèmes aux limites non-linéaires. Dunod, Paris 1969.

[16] J.L. Lions, E. Magenes. Problèmes aux limites non homogênes et applications, Vol. 1, Dunod Paris (1968).

[17] Jesper Lüzen. The prehistory of the theory of distributions, Springer Verlag - N.Y. 1980.

[18] L.A. Medeiros and J. Limaco. On a model for vibrations of elastic membranes. Int. Journal of Differential Equations and Applications, Vol. 2, no 2 (2001) pp. 209-236.

[19] L.A. Medeiros, J. Limaco, S.B. Menezes. Vibrations of elastic strings (Mathematical Aspects), Journal of Computational Analysis and Applications - Part one - Vol. 4 - $\mathrm{n}$ o 2 - April 2002 - pp. 91-127. Part two - Vol. 4 - n⿳ 3 - July 2002 - pp. 211-263.

[20] M. Milla Miranda and L.P. San Gil Jutuca. Existence and boundary stabilization of solutions for Kirchhoff equation. Comm. Partial Differential Equations, 24 (9 \& 10) (1999) pp. 1750-1800.

[21] S.I. Pohozhaev. Quasilinear hyperbolic equation of Kirchhoff type and conservation law. Tr. Mosk. Energ. Inst. Moscow, n⿳ 201 (1974) pp. 118-126.

[22] S.I. Pohozhaev. On a class of quasilinear hyperbolic equations. Math. URSS Sbornik, 25 (1975) pp. $145-158$.

[23] S.I. Pohozhaev. The Kirchhoff quasilinear hyperbolic equation. Differential Equations, Vol. 21, $\mathrm{n}^{\mathrm{O}} 1$ (1985) pp. 101-108 (in Russian).

[24] P.H. Rivera Rodriguez. On nonlinear hyperbolic equations. Rev. de Ciencias, Univ. San Marcos, 74 (1986) pp. 1-16 (Lima-Peru).

[25] H.L. Royden. Real Analysis - Third Edition, Macmilan-N.Y. 1988. 